\title{
THE RIGIDITY PROBLEM FOR ANALYTIC CRITICAL CIRCLE MAPS
}

\author{
D. KHMELEV AND M. YAMPOLSKY
}

\begin{abstract}
It is shown that if $f$ and $g$ are any two analytic critical circle mappings with the same irrational rotation number, then the conjugacy that maps the critical point of $f$ to that of $g$ has regularity $C^{1+\alpha}$ at the critical point, with a universal value of $\alpha>0$. As a consequence, a new proof of the hyperbolicity of the full renormalization horseshoe of critical circle maps is given.
\end{abstract}

Foreword. In the last several decades, since the works of Mostow, Margulis, Sullivan, and others, rigidity problems occupy a central place in the theory of holomorphic dynamical systems. This type of problems is classical in dynamics: a rigidity theorem postulates that in a certain class of dynamical systems equivalence (combinatorial, continuous, smooth, etc.) automatically has a higher regularity. The dynamical systems considered in this paper are critical circle maps, that is smooth homeomorphisms of the circle with a single critical point having a cubic type. These maps have been a subject of intensive study since the early 1980's as one of the two main examples of universality in transition to chaos. In 1984 Yoccoz [Yoc showed that any two such maps with the same irrational rotation number are conjugate by a continuous change of coordinates (this is a generalization of the classical result of Denjoy for $C^{2}$ diffeomorphisms). The central result of this paper is the following rigidity theorem:

Main Theorem. For any two analytic critical circle maps with the same irrational rotation number, the conjugacy, which maps the critical point of one to the critical point of the other is $C^{1+\alpha}$-smooth at the critical point. Here $\alpha>0$ is a universal constant.

The theorem verifies the so-called $C^{1+\alpha}$-rigidity conjecture for critical circle maps. The conjecture has a history dating back to at least the early 1980's, as it is related to the universality in critical circle maps, and together with the similar conjecture for the unimodal maps of the interval it appears in the works of Feigenbaum, Lanford, Sullivan and others. There was some recent progress in the study of the conjecture, as de Faria and de Melo dFdM2 used the methods of McMullen McM2 to establish it for the rotation numbers satisfying the Diophantine condition with the exponent 2, usually referred to in the subject as the bounded type condition. The understanding of the unbounded type

Date: September 8, 2004.

This paper was completed during the authors' stay at the Institut Henri Poincaré during Fall 2003, and presented at the IHP at the same time.

The second author was partially supported by an NSERC Discovery grant. 
requires an analysis of critical circle mappings with an almost parabolic dynamics, which we have carried out in this paper.

\section{Preliminaries}

Some notations. We use dist and diam to denote the Euclidean distance and diameter in $\mathbb{C}$, and we let area $(D)$ denote the area of a domain $D \subset \mathbb{C}$. We shall say that two real numbers $A$ and $B$ are $K$-commensurable for $K>1$ if $K^{-1}|A| \leq|B| \leq K|A|$. We will use the notation $A \underset{K}{\sim} B$ in this case, omitting $K$ and writing simply $A \sim B$ when the commensurability factor is universal. The notation $D_{r}(z)$ will stand for the Euclidean disk with the center at $z \in \mathbb{C}$ and radius $r$. The unit disk $D_{1}(0)$ will be denoted $\mathbb{D}$. The plane $(\mathbb{C} \backslash \mathbb{R}) \cup J$ with the parts of the real axis not contained in the interval $J \subset \mathbb{R}$ removed will be denoted $\mathbb{C}_{J}$. By the circle $\mathbb{T}$ we understand the affine manifold $\mathbb{R} / \mathbb{Z}$, it is naturally identified with the unit circle $S^{1}=\partial \mathbb{D}$. The real translation $x \mapsto x+\theta$ projects to the rigid rotation by angle $\theta$ of the circle, $R_{\theta}: \mathbb{T} \rightarrow \mathbb{T}$. For two points $a$ and $b$ in the circle $\mathbb{T}$ which are not diametrically opposite, $[a, b]$ will denote the shorter of the two arcs connecting them. As usual, $|[a, b]|$ will denote the length of the arc. For two points $a, b \in \mathbb{R},[a, b]$ will denote the closed interval with endpoints $a, b$ without specifying their order. The cylinder in this paper, unless otherwise specified, will mean the affine manifold $\mathbb{C} / \mathbb{Z}$. Its equator is the circle $\{\operatorname{Im} z=0\} / \mathbb{Z} \subset \mathbb{C} / \mathbb{Z}$. A topological annulus $A \subset \mathbb{C} / \mathbb{Z}$ will be called an equatorial annulus, or an equatorial neighborhood, if it has a smooth boundary and contains the equator.

By "smooth" in this paper we will mean "of class $C^{\infty}$ ", unless another degree of smoothness is specified. The notation " $C$ " will stand for "real-analytic".

For a piecewise-analytic dynamical system $\mathcal{F}=\sqcup f_{i}$ in the Riemann sphere we will call the dynamics generated by the iterates of $f_{i}$ and the univalent inverse branches $f_{i}^{-1}$ the complete dynamics of $\mathcal{F}$.

1.1. Statements of the results. A critical circle map is an orientation preserving automorphism of $\mathbb{T}$ of class $C^{3}$ with a single critical point $c$. A further assumption is made that the critical point is of cubic type. This means that for a lift $\bar{f}: \mathbb{R} \rightarrow \mathbb{R}$ of a critical circle map $f$ with critical points at the integer translates of $\bar{c}$,

$$
\bar{f}(x)-\bar{f}(\bar{c})=(x-\bar{c})^{3}(\text { const }+O(x-\bar{c})) .
$$

We note that all the renormalization results will hold true if in the above definition " 3 " as the order of smoothness and the order of the critical point is replaced by any other odd number. To fix our ideas, we will always place the critical point of $f$ at $0 \in \mathbb{T}$.

Being a homeomorphism of the circle, a critical circle map $f$ has a well-defined rotation number, denoted $\rho(f)$. It is useful to represent $\rho(f)$ as a contined fraction with positive 
terms

$$
\rho(f)=\frac{1}{r_{0}+\frac{1}{r_{1}+\frac{1}{r_{2}+\cdots}}}
$$

Further on we will abbreviate this expression as $\left[r_{0}, r_{1}, r_{2}, \ldots\right]$ for typographical convenience. Note that the numbers $r_{i}$ are determined uniquely if and only if $\rho(f)$ is irrational. In this case we shall say that $\rho(f)$ (or $f$ itself) is of the type bounded by $B$ if $\sup r_{i} \leq B$.

The main result of this paper is the following Theorem:

Theorem 1.1. There exists a universal constant $\alpha>0$ such that the following holds. Let $f_{1}$ and $f_{2}$ be two analytic critical circle maps with the same irrational rotation number. Denote $\psi: \mathbb{T} \rightarrow \mathbb{T}$ the conjugacy $\psi \circ f_{1} \circ \psi^{-1}=f_{2}$ fixing the origin. Then $\psi$ is $C^{1+\alpha}$ at the origin.

The above theorem should be seen as a generalization of the result of de Faria and de Melo dFdM1, dFdM2, who showed that when $\rho\left(f_{i}\right)$ is of a type bounded by some constant $B$, the conjugacy $\varphi$ is globally $C^{1+\alpha}$ smooth, with $\alpha=\alpha(B)$. An immediate corrolary is:

Corollary 1.2. The uniform distance between the successive renormalizations $\mathcal{R}^{n} f_{i}$ decreases at a universal geometric rate.

We use this to obtain a new proof of the main result of [Ya4]:

Theorem 1.3. The global renormalization horseshoe of the cylinder renormalization operator $\mathcal{R}_{c y l}$ is uniformly hyperbolic, with one-dimensional unstable direction.

\section{THE GEOMETRY OF THE CLOSEST RETURNS AND RENORMALIZATION}

2.1. The dynamical partition of a critical circle map. Recall that an iterate $f^{k}(0)$ is called a closest return of the critical point if the arc $\left[0, f^{k}(0)\right]$ contains no iterates $f^{i}(0)$ with $i<k$. By a classical result of Poincaré every circle homeomorphism $f$ with an irrational rotation number is semi-conjugate to the rigid rotation $R_{\rho(f)}$. Moreover, Yoccoz Yoc has shown that in the case when $f$ is a critical circle map, the semi-conjugacy becomes a conjugacy, thus extending the classical result of Denjoy to this case. Poincaré's result implies that the order of the points in an orbit of $f$ with an irrational rotation number is the same as that in an orbit of $R_{\rho(f)}$. It follows, in particular, that if we denote $\left\{p_{m} / q_{m}\right\}$ the sequence of best rational approximations of $\rho(f)$ obtained as the truncated continued fractions $p_{m} / q_{m}=\left[r_{0}, r_{1}, \ldots, r_{m-1}\right]$, then the iterates $\left\{f^{q_{m}}(0)\right\}$ are closest returns of 0 . Set $I_{m} \equiv\left[0, f^{q_{m}}(0)\right]$. We will denote $I_{m}^{i}=f^{i}\left(I_{m}\right)$. An important combinatorial fact is that the collection of intervals

$$
\mathcal{P}_{m}=\left\{I_{m}, I_{m}^{1}, \ldots, I_{m}^{q_{m+1}-1}\right\} \cup\left\{I_{m+1}, I_{m+1}^{1}, \ldots, I_{m+1}^{q_{m}-1}\right\}
$$

covers the circle. Moreover, the intervals in the collection $\mathcal{P}_{m}$ may overlap only at the endpoints. Hence we will refer to $\mathcal{P}_{m}$ as the $m$-th dynamical partition of $f$. The geometry 
of the partition (2.1) is essential to our study. Of crucial importance is the following real a priori bound of Świątek and Herman:

Theorem 2.1. There exists a universal constant $K>1$ such that the following holds. Let $f: \mathbb{T} \rightarrow \mathbb{T}$ be a critical circle map with an irrational rotation number. Then there exists $m_{0}=m_{0}(f)$ such that for all $m \geq m_{0}$ and every pair $I, J$ of adjacent atoms of the partition (2.1),

$$
K^{-1}|J| \leq|I| \leq K|J|
$$

Utilizing the above bounds Herman has shown in $\mathrm{He}$ (see dFdM1 for a published account):

Theorem 2.2. Any two critical circle maps with the same irrational rotation numbers are quasisymmetrically conjugate.

2.2. Definition of renormalization of critical circle maps. An analogy with the universality phenomena in statistical physics and with the already discovered FeigenbaumCollett-Tresser universality in unimodal maps, led the authors of FKS and ORSS to explain the existence of the universal constants by introducing a renormalization operator acting on critical circle maps. The definition is by no means straightforward. A detailed discussion may be found in Ya3. We need a supporting definition:

Definition 2.1. A commuting pair $\zeta=(\eta, \xi)$ consists of two $C^{3}$-smooth orientation preserving interval homeomorphisms $\eta: I_{\eta} \rightarrow \eta\left(I_{\eta}\right), \xi: I_{\xi} \rightarrow \xi\left(I_{\xi}\right)$, where

(I) $I_{\eta}=[0, \xi(0)], I_{\xi}=[\eta(0), 0]$;

(II) Both $\eta$ and $\xi$ have homeomorphic extensions to interval neighborhoods of their respective domains with the same degree of smoothness, which commute, $\eta \circ \xi=\xi \circ \eta$;

(III) $\xi \circ \eta(0) \in I_{\eta}$;

(IV) $\eta^{\prime}(x) \neq 0 \neq \xi^{\prime}(y)$, for all $x \in I_{\eta} \backslash\{0\}$, and all $y \in I_{\xi} \backslash\{0\}$.

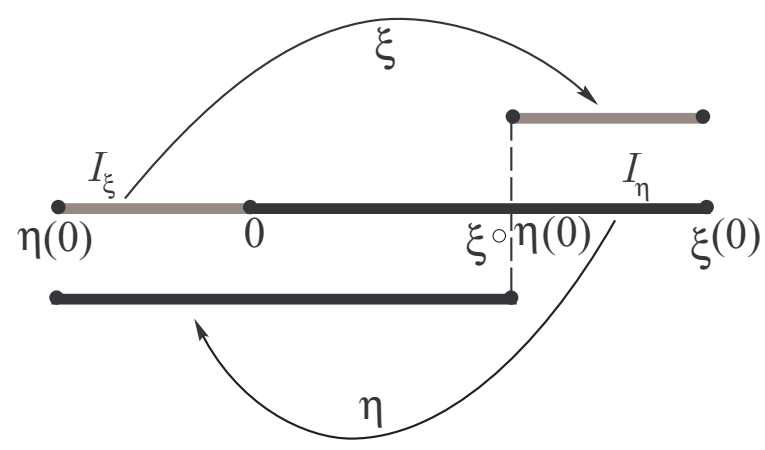

Figure 1. A commuting pair 
The commutation condition allows one to iterate the extensions of the maps of a commuting pair. It also allows us to perform the following glueing construction. Given a critical commuting pair $\zeta=(\eta, \xi)$ we can regard the interval $I=[\eta(0), \xi \circ \eta(0)]$ as a circle, identifying $\eta(0)$ and $\xi \circ \eta(0)$ and define $f_{\zeta}: I \rightarrow I$ by

$$
f_{\zeta}=\left\{\begin{array}{l}
\eta \circ \xi(x) \text { for } x \in[\eta(0), 0] \\
\eta(x) \text { for } x \in[0, \xi \circ \eta(0)]
\end{array}\right.
$$

The mapping $\xi$ extends to a $C^{3}$-diffeomorphism of open neighborhoods of $\eta(0)$ and $\xi \circ \eta(0)$. Using it as a local chart we turn the interval $I$ into a closed one-dimensional manifold $M$. Condition (II) above implies that the mapping $f_{\zeta}$ projects to a well-defined $C^{3}$-smooth homeomorphism $F_{\zeta}: M \rightarrow M$. Identifying $M$ with the circle by a diffeomorphism $\varphi$ : $M \rightarrow \mathbb{T}$ we recover a critical circle mapping $f^{\varphi}=\varphi \circ F_{\zeta} \circ \varphi^{-1}$. The critical circle mappings corresponding to two different choices of $\varphi$ are conjugated by a diffeomorphism, and thus we recovered a $C^{3}$-smooth conjugacy class of circle mappings from a critical commuting pair.

We can metrize the space of $C^{r}$-smooth commuting pairs considered modulo an affine conjugacy as follows (see dFdM1). Let $\zeta_{1}=\left(\eta_{1}, \xi_{1}\right), \zeta_{2}=\left(\eta_{2}, \xi_{2}\right)$ be two such pairs, and denote $w_{i}: \mathbb{C} \rightarrow \mathbb{C}$ a Möbius transformation which maps the ordered triple of points $\eta_{i}(0)$, $0, \xi_{i}(0)$ to $0,1 / 2,1$. The $C^{r}$-distance between $\zeta_{1}$ and $\zeta_{2}$ is set to be

$$
\operatorname{dist}_{C^{r}}\left(\zeta_{1}, \zeta_{2}\right)=\max \left\{\left|\xi_{1}(0) / \eta_{1}(0)-\xi_{2}(0) / \eta_{2}(0)\right|, \operatorname{dist}_{C^{r}}\left(w_{1} \circ \zeta_{1} \circ w_{1}^{-1}, w_{2} \circ \zeta_{2} \circ w_{2}^{-1}\right)\right\} .
$$

Let $f$ be a critical circle mapping, whose rotation number $\rho$ has a continued fraction expansion (1.1) with at least $m+1$ terms, and let $p_{m} / q_{m}=\left[r_{0}, \ldots, r_{m-1}\right]$. The pair of iterates $f^{q_{m+1}}$ and $f^{q_{m}}$ restricted to the circle arcs $I_{m}$ and $I_{m+1}$ correspondingly can be viewed as a critical commuting pair in the following way. Let $\bar{f}$ be the lift of $f$ to the real line satisfying $\bar{f}^{\prime}(0)=0$, and $0<\bar{f}(0)<1$. For each $m>0$ let $\bar{I}_{m} \subset \mathbb{R}$ denote the closed interval adjacent to zero which projects down to the interval $I_{m}$. Let $\tau: \mathbb{R} \rightarrow \mathbb{R}$ denote the translation $x \mapsto x+1$. Let $\eta: \bar{I}_{m} \rightarrow \mathbb{R}, \xi: \bar{I}_{m+1} \rightarrow \mathbb{R}$ be given by $\eta \equiv \tau^{-p_{m+1}} \circ \bar{f}^{q_{m+1}}$, $\xi \equiv \tau^{-p_{m}} \circ \bar{f}^{q_{m}}$. Then the pair of maps $\left(\eta\left|\bar{I}_{m}, \xi\right| \bar{I}_{m+1}\right)$ forms a critical commuting pair corresponding to $\left(f^{q_{m+1}}\left|I_{m}, f^{q_{m}}\right| I_{m+1}\right)$. Henceforth we shall simply denote this commuting pair by

$$
\left(f^{q_{m+1}}\left|I_{m}, f^{q_{m}}\right| I_{m+1}\right) .
$$

This allows us to readily identify the dynamics of the above commuting pair with that of the underlying circle map, at the cost of a minor abuse of notation.

Following $\mathrm{dFdM1}$, we say that the height $\chi(\zeta)$ of a critical commuting pair $\zeta=(\eta, \xi)$ is equal to $r$, if

$$
0 \in\left[\eta^{r}(\xi(0)), \eta^{r+1}(\xi(0))\right]
$$

If no such $r$ exists, we set $\chi(\zeta)=\infty$, in this case the map $\eta \mid I_{\eta}$ has a fixed point. For a pair $\zeta$ with $\chi(\zeta)=r<\infty$ one verifies directly that the mappings $\eta \mid\left[0, \eta^{r}(\xi(0))\right]$ and $\eta^{r} \circ \xi \mid I_{\xi}$ again form a commuting pair. For a commuting pair $\zeta=(\eta, \xi)$ we will denote by $\widetilde{\zeta}$ the pair $\left(\widetilde{\eta}\left|\widetilde{I}_{\eta}, \widetilde{\xi}\right| \widetilde{I}_{\xi}\right)$ where tilde means rescaling by the linear factor $\lambda=-\frac{1}{\left|I_{\eta}\right|}$. 
Definition 2.2. The renormalization of a real commuting pair $\zeta=(\eta, \xi)$ is the commuting pair

$$
\left.\mathcal{R} \zeta=\left(\widetilde{\eta^{r} \circ \xi}\left|\widetilde{I}_{\xi}, \widetilde{\eta}\right|\left[0, \widetilde{\eta^{r}(\xi(0)}\right)\right]\right) .
$$

The non-rescaled pair $\left(\eta^{r} \circ \xi\left|I_{\xi}, \eta\right|\left[0, \eta^{r}(\xi(0))\right]\right)$ will be referred to as the pre-renormalization $p \mathcal{R} \zeta$ of the commuting pair $\zeta=(\eta, \xi)$.

For a pair $\zeta$ we define its rotation number $\rho(\zeta) \in[0,1]$ to be equal to the continued fraction $\left[r_{0}, r_{1}, \ldots\right]$ where $r_{i}=\chi\left(\mathcal{R}^{i} \zeta\right)$. In this definition $1 / \infty$ is understood as 0 , hence a rotation number is rational if and only if only finitely many renormalizations of $\zeta$ are defined; if $\chi(\zeta)=\infty, \rho(\zeta)=0$. Thus defined, the rotation number of a commuting pair can be viewed as a rotation number in the usual sense:

Proposition 2.3. The rotation number of the mapping $F_{\zeta}$ is equal to $\rho(\zeta)$.

There is an advantage in defining $\rho(\zeta)$ using a sequence of heights in removing the ambiguity in prescribing a continued fraction expansion to rational rotation numbers in a renormalization-natural way.

For $\rho=\left[r_{0}, r_{1}, \ldots\right] \in[0,1]$ let us set

$$
G(\rho)=\left[r_{1}, r_{2}, \ldots\right]=\left\{\frac{1}{\rho}\right\}
$$

where $\{x\}$ denotes the fractional part of a real number $x$ ( $G$ is usually referred to as the Gauss map). As follows from the definition,

$$
\rho(\mathcal{R} \zeta)=G(\rho(\zeta))
$$

for a real commuting pair $\zeta$ with $\rho(\zeta) \neq 0$.

The renormalization of the real commuting pair (2.2), associated to some critical circle map $f$, is the rescaled pair $\left(\widetilde{f^{q_{m+2}}}\left|\widetilde{I_{m+1}}, \widetilde{f^{q_{m+1}}}\right| \widetilde{I_{m+2}}\right)$. Thus for a given critical circle map $f$ the renormalization operator recovers the (rescaled) sequence of the first return maps:

$$
\left\{\left(\widetilde{f^{q_{i+1}}}\left|\widetilde{I}_{i}, \widetilde{f^{q_{i}}}\right| \widetilde{I_{i+1}}\right)\right\}_{i=1}^{\infty} .
$$

A critical commuting pair is a commuting pair $(\eta, \xi)$ whose maps are real-analytic. We shall also impose a technical assumption that $\xi$ analytically extends to an interval $(a, b) \ni 0$ with $\xi(a, b) \supset[\eta(0), \xi(0)]$, and has a single critical point 0 in this interval. The space of critical commuting pairs modulo affine conjugacy will be denoted by $\mathbf{C}$; its subset consisting of pairs $\zeta$ with $\chi(\zeta)=\infty$ will be denoted by $\mathbf{S}_{\infty}$. Renormalization is an injective transformation $\mathcal{R}: \mathbf{C} \backslash \mathbf{C}_{\infty} \rightarrow \mathbf{C}$ (see Ya2]).

\section{Holomorphic EXTEnsions OF CRITICAL COMMUTING PAIRS}

In 1986 Eckmann and Epstein [EE] introduced a space of critical commuting pairs now known as the Epstein class. They showed that this class is invariant under the action of $\mathcal{R}$, and constructed the golden-mean fixed point of $\mathcal{R}$ in this class using the methods of geometric complex analysis. It was further shown by various people, such as Sullivan 
(in the unimodal case), Światek, Herman, and Yoccoz, that the renormalizations of any $C^{3}$-smooth commuting pair with an irrational rotation number converge to the Epstein class, at a geometric rate in the $C^{2}$-metric. Below, after some preliminaries, we define the Epstein class, and formulate these results more precisely.

3.1. Carathéodory topology on a space of branched coverings. Consider the collection $\mathbf{X}$ of all triplets $(U, u, f)$, where $U \subset \mathbb{C}$ is a topological disk different from the whole plane, $u \in U$, and $f: U \rightarrow \mathbb{C}$ is a three-fold analytic branched covering map, with the only branch point at $u$. We will topologize $\mathbf{X}$ as follows (cf. [McM1]).

Let $\left\{\left(U_{n}, u_{n}\right)\right\}$ be a sequence of open connected regions $U_{n} \subset \mathbb{C}$ with marked points $u_{n} \in U_{n}$. Recall that this sequence Carathéodory converges to a marked region $(U, u)$ if:

- $u_{n} \rightarrow u \in U$, and

- for any Hausdorff limit point $K$ of the sequence $\hat{\mathbb{C}} \backslash U_{n}, U$ is a component of $\hat{\mathbb{C}} \backslash K$.

For a simply connected $U \subset \mathbb{C}$ and $u \in U$ let $R_{(U, u)}: \mathbb{D} \rightarrow U$ denote the inverse Riemann mapping with normalization $R_{(U, u)}(0)=u, R_{(U, u)}^{\prime}(0)>0$. By a classical result of Carathéodory, the Carathédory convergence of simply-connected regions $\left(U_{n}, u_{n}\right) \rightarrow(U, u)$ is equivalent to the locally uniform convergence of the inverse Riemann mappings $R_{\left(U_{n}, u_{n}\right)}$ to $R_{(U, u)}$.

For positive numbers $\epsilon_{1}, \epsilon_{2}, \epsilon_{3}$ and compact subsets $K_{1}$ and $K_{2}$ of the open unit disk $\mathbb{D}$, let the neighborhood $\mathcal{U}_{\epsilon_{1}, \epsilon_{2}, \epsilon_{3}, K_{1}, K_{2}}(U, u, f)$ of an element $(U, u, f) \in \mathbf{X}$ be the set of all $(V, v, g) \in \mathbf{X}$, for which:

- $|u-v|<\epsilon_{1}$

- $\sup _{z \in K_{1}}\left|R_{(V, v)}(z)-R_{(U, u)}(z)\right|<\epsilon_{2}$,

- and $R_{(U, u)}\left(K_{2}\right) \subset V$, and $\sup _{z \in R_{(U, u)}\left(K_{2}\right)}|f(z)-g(z)|<\epsilon_{3}$.

One verifies that the sets $\mathcal{U}_{\epsilon_{1}, \epsilon_{2}, \epsilon_{3}, K_{1}, K_{2}}(U, u, f)$ form a base of a topology on $\mathbf{X}$, which we will call Carathéodory topology. This topology is clearly Hausdorff, and the convergence of a sequence $\left(U_{n}, u_{n}, f_{n}\right)$ to $(U, u, f)$ is equivalent to the Carathéodory convergence of the marked regions $\left(U_{n}, u_{n}\right) \rightarrow(U, u)$ as well as a locally uniform convergence $f_{n} \rightarrow f$.

3.2. The Epstein class. An orientation preserving interval homeomorphism $g: I=$ $[0, a] \rightarrow g(I)=J$ belongs to the Epstein class $\mathcal{E}$ if it extends to an analytic three-fold branched covering map of a topological disk $G \supset I$ onto the double-slit plane $\mathbb{C}_{\tilde{J}}$, where $\tilde{J} \supset \mathrm{cl} J$. Any map $g$ in the Epstein class can be decomposed as

$$
g=Q_{c} \circ h
$$

where $Q_{c}(z)=z^{3}+c$, and $h: I \rightarrow[0, b]$ is a univalent map $h: G \rightarrow \Delta(h)$ onto the complex plane with six slits, which triple covers $\mathbb{C}_{\tilde{J}}$ under the cubic map $Q_{c}(z)$.

For any $s \in(0,1)$, let us introduce a smaller class $\mathcal{E}_{s} \subset \mathcal{E}$ of Epstein mappings $g: I=$ $[0, a] \rightarrow J \subset \tilde{J}$ for which both $|I|$ and $\operatorname{dist}(I, J)$ are $s^{-1}$-commensurable with $|J|$, the length 
of each component of $\tilde{J} \backslash J$ is at least $s|J|$, and $g^{\prime}(a)>s$. We will often refer to the space $\mathcal{E}$ as the Epstein class, and to each $\mathcal{E}_{s}$ as an Epstein class.

We say that a commuting pair $(\eta, \xi) \in \mathbf{C}$ belongs to the (an) Epstein class if both of its maps do. Similarly, a critical circle map $f$ is Epstein if $\mathcal{R} f$ is in the Epstein class. It immediately follows from the definitions that:

Lemma 3.1. If a renormalizable commuting pair $\zeta$ is in the Epstein class, then the same is true for $\mathcal{R} \zeta$.

Let us make a note of an important compactness property of $\mathcal{E}_{s}$

Lemma 3.2 (Lemma 2.10 Ya2]). Let $s \in(0,1)$. The collection of normalized maps $g \in \mathcal{E}_{s}$ with $I=[0,1]$, with marked domains $(U, 0)$ is sequentially compact with respect to Carathéodory topology.

The importance of the Epstein class lies in the fact that all $C^{1}$-limit points of the sequence $\left\{\mathcal{R}^{m}(f)\right\}_{m=M}^{\infty}$ are in $\mathcal{E}_{s}$ for a universal value of $s>0$. A more precise formulation of this was proved in the recent work of de Faria and de Melo dFdM1]:

Lemma 3.3. There exists a universal constant $s>0$ such that the following holds. Let $f \in C^{r},(r \geq 3)$ be a critical circle map with an irrational rotation number. Then the sequence of real commuting pairs $\mathcal{R}^{m}(f)=\left(\widetilde{f^{q_{m+1}}}\left|\widetilde{I_{m}}, \widetilde{f^{q_{m}}}\right| \widetilde{I_{m+1}}\right)$ is bounded in $C^{r-1}$-metric, and $C^{r-1}$-converges to $\mathcal{E}_{s}$ at a geometric rate. If $f \in C^{\omega}$, then the convergence is uniform on compact subsets of $\mathbb{C}_{[0,1]}$.

In particular, for a critical circle map $f \in \mathcal{E}$ there exists $\sigma>0$ such that all its renormalizations are contained in $\mathcal{E}_{\sigma}$. Moreover, the constant $\sigma$ can be chosen independent on $f$, after skipping the first few renormalizations.

Finally, let us formulate an important statement about critical commuting pairs to be used further in the paper (Lemma 2.13, [Ya2]):

Lemma 3.4 (Parabolic Limits). Let $\zeta=(\eta, \xi) \in \mathcal{E}$ be a critical commuting pair with $\rho(\zeta)=0$, which appears as a limit of a sequence of critical commuting pairs $\left\{\zeta_{n}\right\}$ with $\rho\left(\zeta_{n}\right) \in \mathbb{R} \backslash \mathbb{Q}$. Then the map $\eta$ has a unique fixed point in the interval $I_{\eta}$, which is necessarily parabolic, with multiplier one.

A commuting pair $\zeta=(\eta, \xi) \in \mathcal{E}$ will be called parabolic if the map $\eta$ has a unique fixed point in $I_{\eta}$, which has a unit multiplier; this point will usually be denoted $p_{\eta}$. Note, that by virtue of its uniqueness, $p_{\eta}$ has to be globally attracting on one side for the interval homeomorphism $\left.\eta\right|_{I_{\eta}}$, it is globally attracting on the other side under $\eta^{-1}$.

3.3. Holomorphic commuting pairs. De Faria dF1, dF2 introduced holomorphic commuting pairs to apply the Sullivan's Riemann surface laminations argument to the renormalization of critical circle maps. They are suitably defined holomorphic extensions of critical commuting pairs which replace Douady-Hubbard polynomial-like maps [DH2] A critical commuting pair $\zeta=\left(\left.\eta\right|_{I_{\eta}},\left.\xi\right|_{I_{\xi}}\right)$ extends to a holomorphic commuting pair $\mathcal{H}$ if there exist four simply-connected $\mathbb{R}$-symmetric domains $\Delta, D, U, V$ such that 


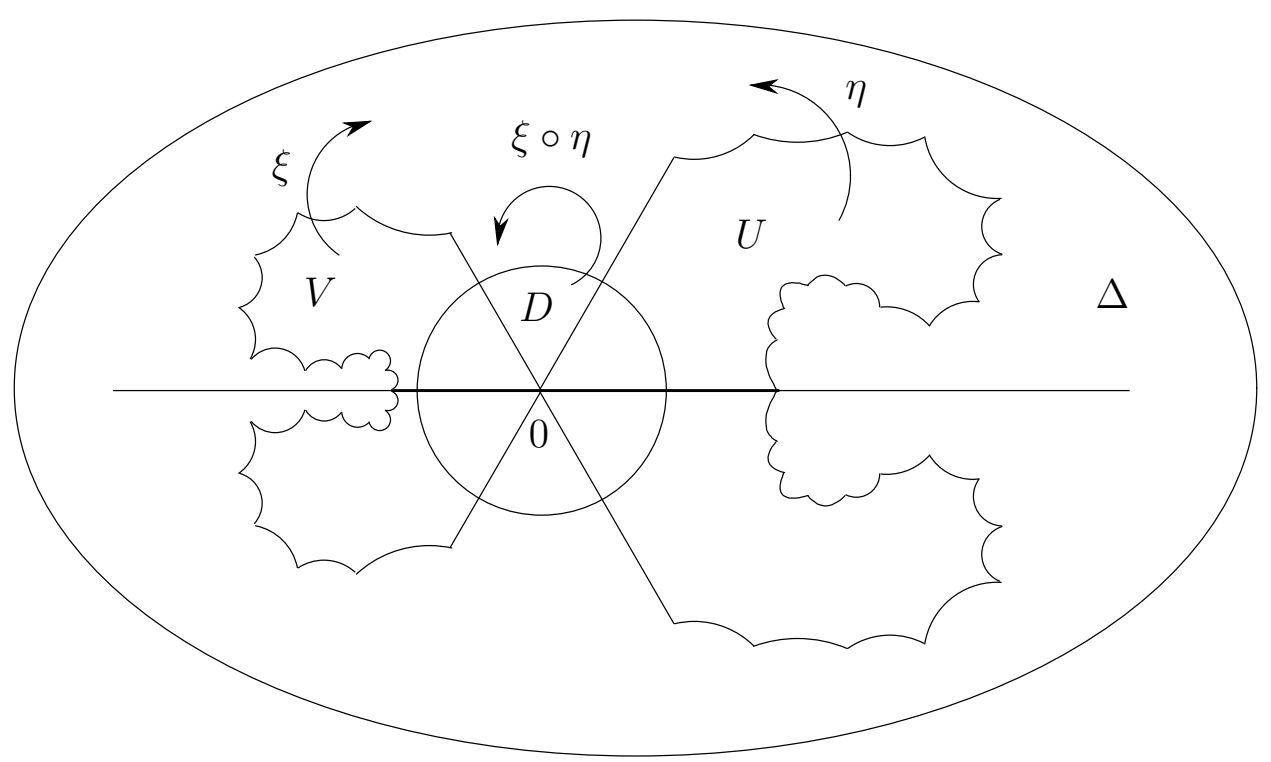

Figure 2. A holomorphic commuting pair

- $\bar{D}, \bar{U}, \bar{V} \subset \Delta, \bar{U} \cap \bar{V}=\{0\}$; the sets $U \backslash D, V \backslash D, D \backslash U$, and $D \backslash V$ are nonempty, connected, and simply-connected, $I_{U}=U \cap \mathbb{R} \supset I_{\eta}, I_{V}=V \cap \mathbb{R} \supset I_{\xi}$;

- mappings $\eta: U \rightarrow(\Delta \backslash \mathbb{R}) \cup \eta\left(I_{U}\right)$ and $\xi: V \rightarrow(\Delta \backslash \mathbb{R}) \cup \xi\left(I_{V}\right)$ are onto and univalent;

- $\nu \equiv \eta \circ \xi: D \rightarrow(\Delta \backslash \mathbb{R}) \cup \nu\left(I_{D}\right)$ is a three-fold branched covering with a unique critical point at zero, where $I_{D}=D \cap \mathbb{R}$.

We shall call $\zeta$ the commuting pair underlying $\mathcal{H}$, and write $\zeta \equiv \zeta_{\mathcal{H}}$. The interval $I_{\mathcal{H}}=[\eta(0), \xi(0)]$ will be called the dynamical interval of $\mathcal{H}$. The domain $D \cup U \cup V$ of a holomorphic commuting pair $\mathcal{H}$ will be denoted $\Omega$ or $\Omega_{\mathcal{H}}$, the range will be denoted $\Delta$ or $\Delta_{\mathcal{H}}$. The closure of the set of points whose orbit under $\mathcal{H}$ is contained in $\Omega$ will be referred to as the filled Julia set of $\mathcal{H}$, denoted $K(\mathcal{H})$. The Julia set of $\mathcal{H}$ is defined as $J(\mathcal{H})=\partial K(\mathcal{H})$

It is easy to see directly from the definition (cf. [dF2]) that:

Proposition 3.5. Let $\zeta$ be a commuting pair with $\chi(\zeta)<\infty$. Suppose $\zeta$ is a restriction of a holomorphic commuting pair $\mathcal{H}$, that is $\zeta=\zeta_{\mathcal{H}}$. Then there exists a holomorphic commuting pair $\mathcal{G}$ with range $\Delta_{\mathcal{H}}$, such that $\zeta_{\mathcal{G}}=\mathcal{R} \zeta$.

The shadow of the holomorphic commuting pair $\mathcal{H}$ is the following piecewise-defined holomorphic dynamical system:

$$
S_{\mathcal{H}}(z)=\left\{\begin{array}{l}
\eta(z), z \in U \\
\xi(z), z \in V \\
\xi \circ \eta(z), z \in D \backslash(U \cup V)
\end{array}\right.
$$


As the next proposition shows one may think of the shadow of a holomorphic commuting pair as an analogue of a cubic-like map:

Proposition 3.6 (Prop. II.4. dF2 ). Given a holomorphic commuting pair $\mathcal{H}$ as above, consider its shadow $S_{\mathcal{H}}$. Let $I=\Omega \cap \mathbb{R}$, and $X=I \cup S_{\mathcal{H}}^{-1}(I)$. Then:

- The restriction of $S_{\mathcal{H}}$ to $\Omega \backslash X$ is a regular three fold covering onto $\Delta \backslash \mathbb{R}$.

- $S_{\mathcal{H}}$ and $\mathcal{H}$ share the same orbits as sets.

We will say that two holomorphic commuting pairs $\mathcal{H}: \Omega_{\mathcal{H}} \rightarrow \Delta_{\mathcal{H}}$ and $\mathcal{G}: \Omega_{\mathcal{G}} \rightarrow \Delta_{\mathcal{G}}$ are conjugate if there is a homeomorphism $h: \Delta_{\mathcal{G}} \rightarrow \Delta_{\mathcal{H}}$ such that

$$
S_{\mathcal{G}}=h^{-1} \circ S_{\mathcal{H}} \circ h \text {. }
$$

In this case we will simply write $\mathcal{G}=h^{-1} \circ \mathcal{H} \circ h$.

3.4. Complex a priori bounds. We shall denote by $\mathbf{H}$ the space of holomorphic commuting pairs $\mathcal{H}: \Omega \rightarrow \Delta$ whose underlying real commuting pair $(\eta, \xi)$ is in the Epstein class. In this case both maps $\eta$ and $\xi$ extend to triple branched coverings $\hat{\eta}: \hat{U} \rightarrow \Delta \cap \mathbb{C}_{\eta\left(J_{\eta}\right)}$ and $\hat{\xi}: \hat{V} \rightarrow \Delta \cap \mathbb{C}_{\xi\left(J_{\xi}\right)}$ respectively. We will turn $\mathbf{H}$ into a topological space by identifying it with a subset of $\mathbf{X} \times \mathbf{X}$ by $\mathcal{H} \mapsto(\hat{U}, 0, \hat{\eta}) \times(\hat{V}, 0, \hat{\xi})$ (cf. \$3.1).

We say that a real commuting pair $(\eta, \xi)$ with an irrational rotation number has complex a priori bounds, if all its renormalizations extend to holomorphic commuting pairs with bounded moduli:

$$
\bmod (\Delta \backslash \Omega)>\mu>0
$$

For $\mu \in(0,1)$ let $\mathbf{H}(\mu)$ denote the space of holomorphic commuting pairs $\mathcal{H}: \Omega_{\mathcal{H}} \rightarrow \Delta_{\mathcal{H}}$, $\operatorname{with} \bmod \left(\Delta_{\mathcal{H}} \backslash \Omega_{\mathcal{H}}\right)>\mu, \min \left(\left|I_{\eta}\right|,\left|I_{\xi}\right|\right)>\mu$ and $\operatorname{diam}\left(\Delta_{\mathcal{H}}\right)<1 / \mu$.

Lemma 3.7 (Lemma 2.15 Ya2]). For each $\mu \in(0,1)$ the space $\mathbf{H}(\mu)$ is sequentially precompact, with every limit point contained in $\mathbf{H}(\mu / 2)$.

The existense of complex a priori bounds is a key analytic issue of renormalization theory. In the case of critical circle maps it is settled by the following theorem:

Theorem 3.8. There exist universal constants $\mu>0$ and $K>1$ such that the following holds. Let $\zeta \in \mathbf{C}$ be a critical commuting pair with an irrational rotation number. Then there exists $N=N(\zeta)$ such that for all $n \geq N$ the commuting pair $\mathcal{R}^{n} \zeta$ extends to a holomorphic commuting pair $\mathcal{H}_{n}: \Omega_{n} \rightarrow \Delta_{n}$ in $\mathbf{H}(\mu)$. The range $\Delta_{n}$ is a Euclidean disk of radius at most $K$, and the regions $\Omega_{n} \cap( \pm \mathbb{H})$ are $K$-quasidisks.

Remark 3.1. We first proved this theorem in Ya1 for commuting pairs $\zeta$ in an Epstein class $\mathcal{E}_{s}$, in which case $N=N(s)$. Our proof was later adapted by de Faria and de Melo dFdM2 to the case of a non-Epstein critical commuting pair. In the general case, in a Carathéodory compact family of critical commuting pairs, the number $N$ can be chosen uniformly. 
Let $\zeta$ be at least $n$ times renormalizable critical commuting pair. For the lack of a better term, let us say that the pair of numbers $\tau_{n}(\zeta)=\left(r_{n-1}, r_{n-2}\right)$ forms the history of the pair $\mathcal{R}^{n} \zeta$. Based on the above theorem and a detailed analysis of the shapes of the domains $\Omega_{n}$ we proved the following in [Ya1]:

Theorem 3.9 ([Ya1]). There exists a universal constant $K_{1}>1$ such that the following holds. Let $\zeta_{1}=\left(\eta_{1}, \xi_{1}\right)$ and $\zeta_{2}=\left(\eta_{2}, \xi_{2}\right)$ be two critical commuting pairs with irrational rotation numbers. Let $n>\max \left(N\left(\zeta_{1}\right), N\left(\zeta_{2}\right)\right)+1$ as above. Assume that the $n$-th renormalizations of $\zeta_{1}, \zeta_{2}$ have the same rotation number and the same history. Then their holomorphic commuting pair extensions $\mathcal{H}_{n}^{1}, \mathcal{H}_{n}^{2}$ are $K_{1}$-quasiconformally conjugate. The conjugating map is conformal on the filled Julia set.

For commuting pairs of the type bounded by $B$ this theorem was first proved by de Faria dF1, dF2, with " $K_{1}$ " depending on the value of $B$. The proof of the above theorem in the case of an unbounded type rotation number requires an analysis of the shape of the domain of the holomorphic pair. In what follows, let $f$ be an analytic critical circle mapping with $\rho(f) \in \mathbb{R} \backslash \mathbb{Q}$, fix a sufficiently large $n$ and let $\mathcal{H}: \Omega \rightarrow \Delta$ be the holomorphic pair extension of $\mathcal{R}^{n} f$ guaranteed by Theorem 3.8 .

Consider the inverse orbit:

$$
J_{0} \equiv f^{q_{n+1}}\left(I_{n}\right), J_{-1} \equiv f^{q_{n+1}-1}\left(I_{n}\right), \ldots, J_{-\left(q_{n+1}-1\right)} \equiv f\left(I_{n}\right),
$$

and the corresponding inverse orbit for the domain $\Delta_{0} \equiv \Delta \cap \mathbb{H}$ :

$$
\Delta_{0}, \Delta_{-1}, \ldots, \Delta_{q_{n+1}-(-1)} \equiv f(U) \cap \mathbb{H} .
$$

Consider the consecutive returns of the orbit (3.2) to $I_{m-1}$ before the first return to $I_{m}$,

$$
J_{-q_{m}}, J_{-2 q_{m}}, \ldots, J_{-l_{m} q_{m}} \text {. }
$$

Consider the curve segment $\gamma^{m} \subset f^{-q_{m}}\left(\left[f^{q_{m-1}-q_{m}}(0), f^{q_{m-1}}(0)\right]\right) \cap \mathbb{H}, \mathrm{cl} \gamma^{m} \ni f^{q_{m-1}-q_{m}}(0)$, and let

$$
\gamma^{m} \equiv \gamma_{-1}^{m}, \gamma_{-2}^{m}, \ldots, \gamma_{-l_{m}}^{m}
$$

be the corresponding inverse orbit of the curve segment $\gamma^{m}$ under $f^{q_{m}}$. Let the curve $\Gamma^{m}$ be the union of the segments $\gamma_{i}^{m}$, for $i=-1, \ldots,-l_{m}$.

Lemma 3.10. There exists a topological disk $\hat{D} \subset D\left(\left[f^{s q_{n}-q_{n+1}}(0), f^{-q_{n-1}-s q_{n}}(0)\right]\right)$ commensurable with $I_{n-1}$, such that

- The domain $U \backslash \hat{D}$ is a $K$-quasidisk for some fixed $K$.

- The intersection $\partial U \cap \hat{D} \cap \mathbb{H}$ is contained in the curve $f^{-\left(q_{n-1}-1\right)}\left(\Gamma^{n}\right)$, where $\Gamma^{n}$ is as above.

In conclusion, let us say that a holomorphic pair $\mathcal{H}$ is $K$-bounded if the conclusions of Theorem 3.8 and Lemma 3.10 hold for $\mathcal{H}$ with this value of $K$ and $\mu=1 / K$, and each of the maps $\xi, \eta$, and $\nu$ constituting $\mathcal{H}$ is a composition of the cubic map $z \mapsto z^{3}$ and a conformal diffeomorphism with $K$-bounded distortion. 


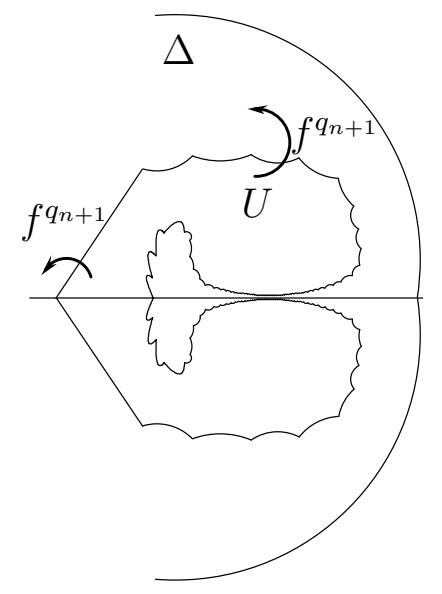

FIGURE 3.

\section{MCMULLEN'S Rigidity RESUlT}

We are going to briefly review here some of the results of McMullen on geometric limits in dynamics, as discussed in Chapter 9 of [McM2]. The definitions are at times quite technical, therefore we will not attempt to go into every detail, and will instead try to outline the main points relevant to our analysis. McMullen gives a general definition of a holomorphic dynamical system $\mathcal{F}$ in $\hat{\mathbb{C}}$ as a countable collection of analytic hypersurfaces in $\hat{\mathbb{C}} \times \hat{\mathbb{C}}$ which define the holomorphic relationships forming $\mathcal{F}$. He endows the space of holomorphic dynamical systems with the Hausdorff topology, the convergence in this topology is referred to as the geometric convergence. Following dFdM2, we will consider the holomorphic dynamical system $\mathcal{F}=\mathcal{F}(\mathcal{H})$ generated by the complete dynamics of a holomorphic pair $\mathcal{H}$. Each hypersurface in $\mathcal{F}(\mathcal{H})$ is the graph of a relation $\left(S_{\mathcal{H}}\right)^{i}(z)=\left(S_{\mathcal{H}}\right)^{j}(z)$ for some $i, j \in \mathbb{Z}$.

Recall that a line field $\mu$ on $\hat{\mathbb{C}}$ is parabolic if $\mu=A^{*}(d z / d \bar{z})$ where $A \in \operatorname{Aut}(\hat{\mathbb{C}})$. A dynamical system $\mathcal{F}$ is nonlinear if no parabolic line field is left invariant by $\mathcal{F}$. It is twisting if any holomorphic dynamical system $\mathcal{F}_{1}$ which is quasiconformally conjugate to $\mathcal{F}$ is non-linear. A quantitative measure of non-linearity may be introduced as follows. Let

$$
\sigma(z)|d z|=\frac{2|d z|}{1+|z|^{2}}
$$

be the spherical metric and denote $B(x, r)$ the spherical ball of radius $r$ centered at $x$. Let $B_{1}, \ldots, B_{k}$ be any collection of disjoint spherical balls, and let $f_{i}: B_{i} \rightarrow \hat{\mathbb{C}}$ be a univalent branch in $\mathcal{F}$ which extends to a ball with twice the radius. The nonlinearity $\nu(\mathcal{F})$ of $\mathcal{F}$ is defined as

$$
\nu(\mathcal{F})=\inf _{\text {parabolic } \mu} \sup _{\text {collections } f_{1}, \ldots, f_{k}} \sum_{i=1}^{k} \int_{B_{i}}\left|\mu-\left(f_{i}\right)^{*} \mu\right| \sigma^{2}(z) d x d y
$$


Our definition varies slightly form the definition of [McM2] who considers supremum over the collections of maps with only a single element. It is not difficult to see that this change does not affect the conclusions. As an example, the reader is invited to observe how the proof of Lemma 9.12 of [McM2] will look with our definition (this lemma is a key element of the proof of the Inflexibility Theorem below). Instead of passing to the limit of a subsequence $\mathcal{F}_{n}^{\text {sat }} \rightarrow \mathcal{G}$ as McMullen does, we consider a blow up $v_{n}$ of $v$, which is nearly invariant under a uniformly twisting system $\mathcal{G}_{n}$ in the measurable sense. This leads to a contradiction in the same way as before.

For a holomorphic dynamical system $\mathcal{F}$ and $K>1$ set

$$
\nu^{K}(\mathcal{F})=\inf _{\varphi} \nu\left(\varphi_{*}(\mathcal{F})\right)
$$

where infinum is taken over $K$-quasiconformal mappings $\varphi: \hat{\mathbb{C}} \rightarrow \hat{\mathbb{C}}, \varphi(0)=0, \varphi(1)=1$, $\varphi(\infty)=\infty$. A collection of holomorphic dynamical systems $\mathcal{F}_{\alpha}$ is uniformly twisting if for any $K>1$

$$
\inf _{\alpha} \nu^{K}\left(\mathcal{F}_{\alpha}\right)>0
$$

A holomorphic commuting pair $\mathcal{H}$ is uniformly twisting if any collection of blow-ups of $\mathcal{F}=\mathcal{F}(\mathcal{H})$ at the points of $J(\mathcal{H})$ is uniformly twisting. More precisely, let $\Lambda(\mathcal{H})$ be the set of all pairs $(x, r) \in \mathbb{C} \times \mathbb{R}_{+}$such that $x \in J(\mathcal{H}), 0 \leq r \leq 1$. Let

$$
\nu^{K}(\mathcal{F}, \Lambda(\mathcal{H}))=\inf _{\omega \in \operatorname{ch}(\Lambda(\mathcal{H}))} \nu^{K}\left(T_{*}^{\omega}(\mathcal{F})\right)
$$

where $\operatorname{ch}(\Lambda(\mathcal{H}))$ is the convex hull of $\Lambda$ in $\mathbb{C} \times \mathbb{R}_{+}$and $T^{\omega}, \omega=(x, r)$ is the fractional linear transformation moving the ball $B(x, r)$ to $B(0,1)$. Then $\mathcal{H}$ is called uniformly twisting if $\nu^{K}(\mathcal{F}, \Lambda(\mathcal{H}))>0$ for all $K>1$.

The relevance of the above definitions lies in the rigidity result below; to formulate it we need another definition. A point $x \in J(\mathcal{H})$ is a $\beta$-deep point if there exists $\beta>0$ such that for every spherical ball $B(x, r)$ with $r$ sufficiently small, the largest ball contained in $B(x, r) \backslash J(\mathcal{H})$ has radius $s(r) \leq r^{1+\beta}$.

Theorem 4.1 (Dynamical inflexibility McM2]). Let $(\mathcal{F}(\mathcal{H}), \Lambda(\mathcal{H}))$ be uniformly twisting and let $\varphi: \hat{\mathbb{C}} \rightarrow \hat{\mathbb{C}}$ be a $K$-quasiconformal conjugacy between holomorphic pairs $\mathcal{H}$ and $\mathcal{G}$. Then for any $\beta$-deep point $x$ of $J(\mathcal{H}), \varphi$ is $C^{1+\alpha}$ conformal at $x$ and constant $\alpha$ depends only on $K, \delta$ and $\nu^{K}(\mathcal{F}(\mathcal{H}), \Lambda(\mathcal{H}))$.

de Faria and de Melo showed (see $\mathrm{dFdM2}$ ):

Theorem 4.2. Let $\mathcal{H}$ be a holomorphic commuting pair with an irrational rotation number $\rho$. There exists $\beta>0$ such that the following holds:

(I) the critical point 0 is a $\beta$-deep point of $J(\mathcal{H})$;

(II) if $\rho$ is of bounded type, then $(\mathcal{F}(\mathcal{H}), J(\mathcal{H}))$ is uniformly twisting.

To prove part (II) of Theorem 4.2 de Faria and de Melo show: 
Lemma 4.3 (dFdM2 $)$. For every $A \in \mathbb{N}$ there exist constants $K>1, \delta>0, \mu>0$, $C>0$ such that for any holomorphic pair $\mathcal{H}$ with an irrational rotation number of a type bounded by $A$, for every point $z \in J(\mathcal{H})$, and every $0<r<\delta$, the disk $B(z, r)$ contains a holomorphic pair $\mathcal{H}_{1}: \Omega_{1} \rightarrow \Delta_{1} \in \mathcal{F}(\mathcal{H})$ with the complex a priori bound $\mu$, such that $\operatorname{diam}\left(\Delta_{1}\right)>C r$, and $\Delta_{1}$ is a $K$-quasidisk.

They then use the following fact:

Proposition 4.4 ( $\mathrm{dFdM2}$ ). In the notation of the previous lemma, there exists $v>0$ depending only on the values of $K, \mu$, and $C$, such that the nonlinearity of $S_{\mathcal{H}_{1}}$ is bounded below by $v \cdot \operatorname{area}\left(\Delta_{1}\right)$

Taking the branches of $S_{\mathcal{H}_{1}}$ to be the collection of maps in (4.1), we see that the nonlinearity of $\mathcal{F}$ in the disk $B(z, r)$ is bounded from below by const - area $(B(z, r))$. After rescaling $B(z, r)$ to $B(0,1)$, we see that the nonlinearity is bounded below by a uniform constant, and hence $\mathcal{H}$ is uniformly twisting.

In this paper we will demonstrate:

Theorem 4.5. There exists $\nu_{0}>0$ such that for every holomorphic pair $\mathcal{H}$ with an irrational $\rho,(\mathcal{F}(\mathcal{H}), J(\mathcal{H}))$ is uniformly twisting, with

$$
\nu^{K_{1}}(\mathcal{F}(\mathcal{H}), \Lambda(\mathcal{H})) \geq \nu_{0}
$$

where $K_{1}$ is as in Theorem 3.9

To understand the difficulty involved in proving uniform twisting in the case of a rotation number of unbounded type, we need first to discuss the local theory of parabolic perturbations. After a brief discussion, in the next section we will outline the idea of the proof of Theorem 4.5.

\section{PARABolic MAPs AND Their Perturbations}

5.1. General facts. We begin with a brief review of the theory of parabolic bifurcations, as applied in particular to an interval map in the Epstein class. For a more comprehensive exposition the reader is referred to [Do], supporting technical details may be found in [Sh]. Fix a map $\eta_{0} \in \mathcal{E}$ having a parabolic fixed point $p$ with unit multiplier.

Theorem 5.1 (Fatou Coordinates). There exist topological discs $U^{A}$ and $U^{R}$, called attracting and repelling petals, whose union is a punctured neighborhood of the parabolic periodic point $p$ such that

$$
\begin{gathered}
\eta_{0}\left(\bar{U}^{A}\right) \subset U^{A} \bigcup\{p\}, \text { and } \bigcap_{k=0}^{\infty} \eta_{0}^{k}\left(\bar{U}^{A}\right)=\{p\}, \\
\eta_{0}\left(\bar{U}^{R}\right) \subset U^{R} \bigcup\{p\}, \text { and } \bigcap_{k=0}^{\infty} \eta_{0}^{-k}\left(\bar{U}^{R}\right)=\{p\},
\end{gathered}
$$

where $\eta_{0}^{-1}$ is the univalent branch fixing $\zeta$. 
Moreover, there exist injective analytic maps

$$
\Phi^{A}: U^{A} \rightarrow \mathbb{C} \text { and } \Phi^{R}: U^{R} \rightarrow \mathbb{C},
$$

unique up to post-composition by translations, such that

$$
\Phi^{A}\left(\eta_{0}(z)\right)=\Phi^{A}(z)+1 \text { and } \Phi^{R}\left(\eta_{0}(z)\right)=\Phi^{R}(z)-1 .
$$

The Riemann surfaces $C^{A}=U^{A} / \eta_{0}$ and $C^{R}=U^{R} / \eta_{0}$ are conformally equivalent to the cylinder $\mathbb{C} / \mathbb{Z}$.

The coordinate change $\Phi^{A}(z)=-1 /(z-p)+o(1 /(z-p))$ and similarly for $\Phi^{R}$.

We denote $\pi_{A}: U^{A} \rightarrow C^{A}$ and $\pi_{R}: U^{R} \rightarrow C^{R}$ the natural projections. The quotients $C^{A}$ and $C^{R}$ are customarily referred to as Écalle-Voronin cylinders; we will find it useful to regard these as Riemann spheres with distinguished points,+- filling in the punctures. The real axis projects to the natural equators $E^{A} \subset C^{A}$ and $E^{R} \subset C^{R}$. Any conformal transit homeomorphism $\tau: C^{A} \rightarrow C^{R}$ fixing the ends,+- is a translation in suitable coordinates. Lifiting it produces a map $\bar{\tau}: U^{A} \rightarrow \mathbb{C}$ satisfying

$$
\tau \circ \pi_{A}=\pi_{R} \circ \bar{\tau} .
$$

We will sometimes write $\tau \equiv \tau_{\theta}$, and $\bar{\tau}=\bar{\tau}_{\theta}$, where

$$
\Phi^{R} \circ \bar{\tau} \circ\left(\Phi^{A}\right)^{-1}(z) \equiv z+\theta \bmod \mathbb{Z} .
$$

The return map from $U^{R}$ to $U^{A}$ descends to a well-defined analytic transformation

$$
\mathcal{E}: \mathcal{W} \rightarrow C^{A}
$$

(the Écalle-Voronin map) where $\mathcal{W}$ is an open subset of $C^{R}$. It is easy to see that the ends of $C^{R}$ belong to different components of $\mathcal{W}$. The choice of a conformal transit isomorphism

$$
\Theta: C^{A} \rightarrow C^{R}
$$

respecting these ends determines an analytic dynamical system

$$
\mathcal{F}_{\Theta}=\Theta \circ \mathcal{E}: \mathcal{W} \rightarrow C^{R}
$$

with fixed points at \pm . The product of the corresponding eigenvalues $\varrho_{\Theta}^{ \pm}$is clearly independent of $\Theta$, and by the Schwarz Lemma is a number greater than one.

Suppose for an analytic map $\eta$ in a sufficiently small neighborhood of $\eta_{0}$ the parabolic point splits into a complex conjugate pair of repelling fixed points $p_{\eta} \in \mathbb{H}$ and $\bar{p}_{\eta}$ with multipliers $\lambda_{\eta}^{ \pm}=e^{2 \pi i \pm \alpha(\eta)}$. In this situation one may still speak of attracting and repelling petals:

Lemma 5.2 (Douady Coordinates). Let $V \subset \mathbb{C}$ be a domain containing $I_{\eta_{0}}$. There exists a Carathéodory neighborhood $U\left(\eta_{0}\right)$ of the map $\eta_{0}$ in the domain $V$ such that the following holds. For any $\eta \in U\left(\eta_{0}\right)$ with $|\arg \alpha(\eta)|<\pi / 4$, there exist topological discs $U_{\eta}^{A}$ and $U_{\eta}^{R}$ whose union is a neighborhood of $p$, and injective analytic maps

$$
\Phi_{\eta}^{A}: U^{A} \rightarrow \mathbb{C} \text { and } \Phi_{\eta}^{R}: U_{f}^{R} \rightarrow \mathbb{C}
$$


unique up to post-composition by translations, such that

$$
\Phi_{\eta}^{A}(\eta(z))=\Phi_{\eta}^{A}(z)+1 \text { and } \Phi_{\eta}^{R}(\eta(z))=\Phi_{\eta}^{R}(z)+1 .
$$

The quotients $C_{\eta}^{A}=U_{\eta}^{A} / \eta$ and $C_{\eta}^{R}=U_{\eta}^{R} / \eta$ are Riemann surfaces conformally equivalent to $\mathbb{C} / \mathbb{Z}$.

Let us note:

Proposition 5.3. There exists an open neighborhood $\mathcal{W}\left(\eta_{0}\right)$ of $\eta_{0}$ in the Carathéodory topology in the domain $V$, such that for every $\eta \in \mathcal{W}\left(\eta_{0}\right)$ as above, the condition on the eigenvalues of the repelling fixed points is automatically satisfied.

An arbitrary choice of real basepoints $a \in U^{A}$ and $r \in U^{R}$ enables us to specify the Fatou and Douady coordinates uniquely, by requiring that $\Phi^{A}(a)=\Phi_{\eta}^{A}(a)=0$, and $\Phi^{R}(r)=\Phi_{\eta}^{R}(r)=0$. The following fundamental theorem first appeared in [DH1]:

Theorem 5.4. With these normalizations the maps $\Phi_{\eta}^{A}, \Phi_{\eta}^{R}$ depend continuously on $\eta$ with respect to the compact-open topology, and

$$
\Phi_{\eta}^{A} \rightarrow \Phi^{A} \text { and } \Phi_{\eta}^{R} \rightarrow \Phi^{R}
$$

uniformly on compact subsets of $U^{A}$ and $U^{R}$ respectively.

Moreover, select the smallest $n(\eta) \in \mathbb{N}$ for which $\eta^{n(\eta)}(a) \geq r$. Then

$$
\eta^{n(\eta)}(z)=\left(\Phi_{\eta}^{R}\right)^{-1} \circ T_{\theta(\eta)+K} \circ \Phi_{\eta}^{A}
$$

wherever both sides are defined. In this formula $T_{a}(z)$ denotes the translation $z \mapsto z+a$, $\theta(\eta) \in[0,1)$ is given by

$$
\theta(\eta)=1 / \alpha(\eta)+\underset{\alpha(\eta) \rightarrow \infty}{o(1)} \bmod 1
$$

and the real constant $K$ is determined by the choice of the basepoints a, $r$. Thus for a sequence $\left\{\eta_{k}\right\} \subset U(\eta)$ converging to $\eta$, the iterates $\eta_{k}^{n\left(\eta_{k}\right)}$ converge locally uniformly if and only if there is a convergence $\theta(\eta) \rightarrow \theta$, and the limit in this case is a certain lift of the transit homeomorphism $\tau_{\theta}$ for the parabolic map $\eta_{0}$.

5.2. An explanation of the method of the proof of Theorem 4.5. Let us begin by explaining the difference between the bounded and unbounded cases. To understand what happens in the case of an unbounded type, consider a sequence of renormalizable holomorphic pairs $\mathcal{H}_{n} \rightarrow \mathcal{H}$ with $\rho(\mathcal{H})=0$, such that $\mathcal{R}\left(\mathcal{H}_{n}\right)$ also converge to a holomorphic pair $\mathcal{H}^{\prime}$. In the Fatou coordinates of $\mathcal{H}$, the preimages of of $\mathcal{H}^{\prime}$ form a grid $\Lambda$, which up to a bounded distortion is produced by the translations $z \mapsto z+1, z \mapsto z+\log \left(\mathcal{E}^{\prime}(+)\right) / 2 \pi i$ (cf. Figure 4).

Since the Fatou coordinates have the order $1 /(z-p)$, the size of the largest holomorphic commuting pair of the grid in the disk $D_{r}(p)$ is of the order $r^{2}$. Thus in this case we cannot get the uniform twisting condition by finding a single holomorphic pair commensurable 


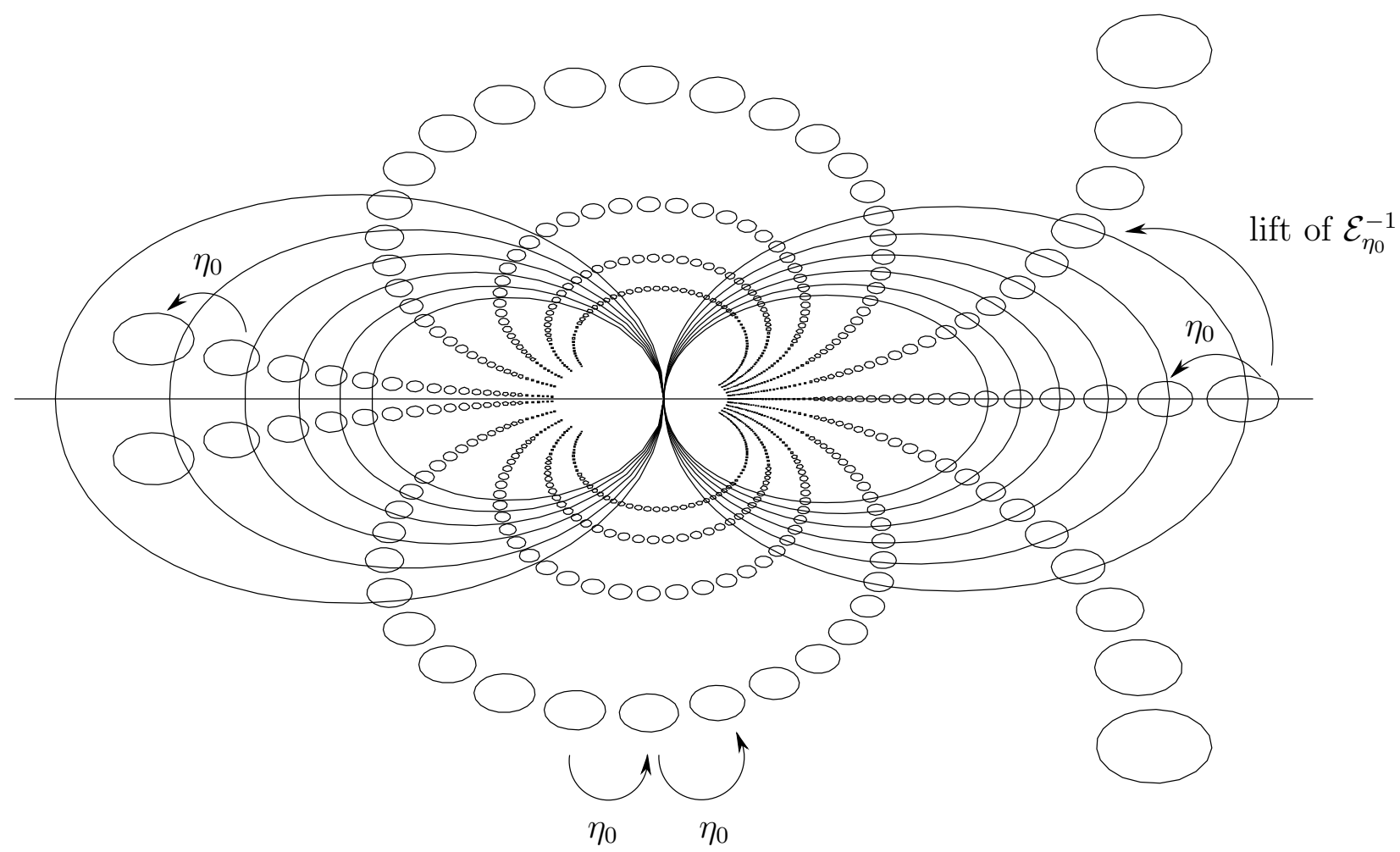

FiguRE 4. Images of a disk under the parabolic dynamics

with the disk, as in Lemma 4.3. This is precisely where the argument of dFdM2 fails in the case of unbounded type.

The problem is resolved in this paper using the following simple idea. In any disk in $\mathbb{C}$, the domains of the holomorphic pairs of the grid $\Lambda$ take up a universally bounded proportion of the area. Therefore, the same is true in each of the disks $D_{r}(p)$ for small enough $r$. We will thus use all of the copies of $\mathcal{H}^{\prime}$ rather than just one of them to prove that $\mathcal{H}$ is uniformly twisting.

\section{KEY TECHNICAL LEMMA}

For a critical commuting pair $(\eta, \xi)$ set $I=I_{\eta} \backslash \eta\left(I_{\eta}\right)$. Notice that the largest $r$ for which $\eta^{r}(I) \subset I_{\eta}$ is equal to the height $\chi(\zeta)$.

Lemma 6.1. For every $K>1$ there exists $0<C<1$ such that the following holds. Suppose $\zeta$ is a critical commuting pair with an irrational rotation number. Then there exists $M$ such that for every $m>M$ denoting $(\eta, \xi)=\mathcal{R}^{m} \zeta$ and $r=\chi(\eta, \xi)$, we have the following.

Let $\Delta$ be a disk $K$-commensurable with one of the intervals $\eta^{\varkappa}(I), 0 \leq \varkappa \leq r$, such that $\Delta$ is symmetric with respect to the real axis, $\Delta \cap \eta^{\varkappa}(I) \neq \varnothing$ and $\left(\Delta \backslash \eta^{\varkappa}(I)\right) \cap \mathbb{R}=\varnothing$, 


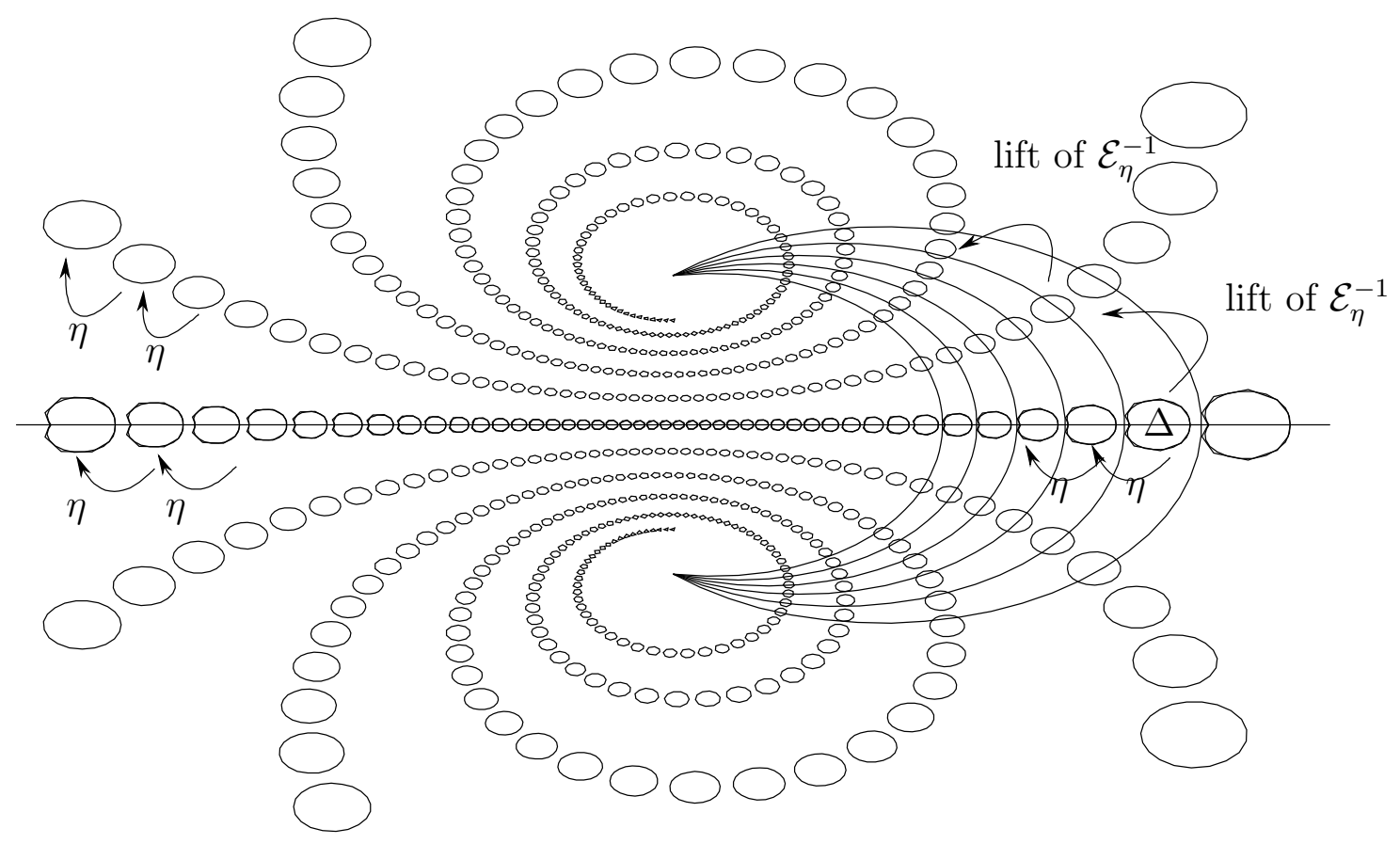

FiguRE 5. Images of disk $\Delta$ under the full dynamics of $\eta$

$\bmod (\Delta) \geq 1 / K>0$. Then for any $z_{0} \in \eta^{i}(I), 0 \leq i \leq r$ for any $l \in\left[\left|\eta^{i}(I)\right|, 1\right]$ there exists a pointed area $(U, y)$ such that $\left|z_{0}-y\right| \sim l$, $\operatorname{diam} U \sim l$ and

$$
\sum_{\tilde{\Delta} \subset U} \operatorname{area}(\tilde{\Delta}) \geq C l^{2}
$$

where $\varphi \tilde{\Delta}=\Delta$ for some $\varphi$ from the complete dynamics generated by $\eta$.

Proof. It is instructive to have a look at Figure [5, to see how $\Delta$ is moved around under $\eta$ and $\eta^{-1}$. Notice that the mapping $\mathcal{E}_{\eta}$ is some pertubation of Ecalle-Voronin mapping $\mathcal{E}$. In fact, $\mathcal{E}_{\eta}$ is the return map $R_{\eta}$ to a strip connecting $z_{-}$to $z_{+}$. To describe the picture mathematically, we have to pass to the Douady coordinates. First, Theorem 3.8 guarantees that there exists $M>0$ such that for all $m>M$ commuting pair $\mathcal{R}^{m} \zeta$ extends to a holomorphic commuting pair $\mathcal{H}_{m}$ with universal complex a priori bounds. Hence we can apply the following fact, which follows from Lemma 5.2 and Theorem 5.4 .

There exist universal $\delta>0, N>0$, such that for all $r>N$ the following statements hold. Denote $\Phi_{\eta}^{A}$ and $\Phi_{\eta}^{R}$ the attracting and repelling Douady coordinates of $\eta$, defined in $U^{A}$ and $U^{R}$ respectively, where $U^{A}$ and $U^{R}$ are real-symmetric disks of radius $\delta$ whose boundaries contain both of the points $z_{+}, z_{-}$.

For $\tau_{-}(z)=z_{-}-1 /\left(z-z_{-}\right)$and $\tau_{+}(z)=z_{+}-1 /\left(z-z_{+}\right)$we have

$$
\frac{1}{K} \leq\left(\tau_{-} \circ\left(\Phi_{\eta}^{A}\right)^{-1}\right)^{\prime}(w) \leq K \text { for } w \in \Phi_{\eta}^{A}\left(U^{A} \cap(-\mathbb{H})\right)
$$




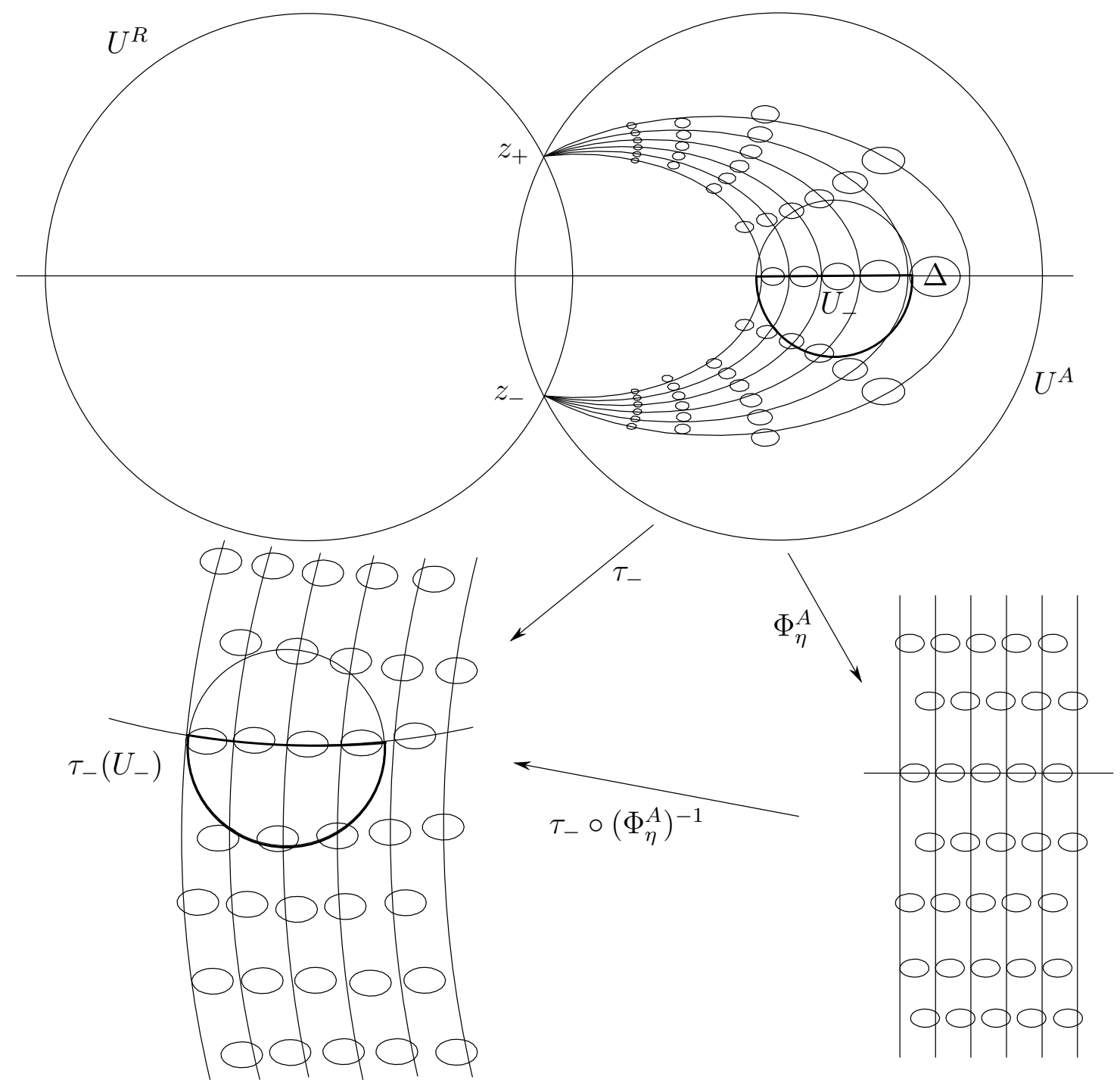

FiguRE 6. Estimate for the area of discs $\tilde{\Delta}$, covered by $U_{-}$

$$
\begin{gathered}
\frac{1}{K} \leq\left(\tau_{+} \circ\left(\Phi_{\eta}^{A}\right)^{-1}\right)^{\prime}(w) \leq K \text { for } w \in \Phi_{\eta}^{A}\left(U^{A} \cap \mathbb{H}\right), \\
\frac{1}{K} \leq\left(\tau_{-} \circ\left(\Phi_{\eta}^{R}\right)^{-1}\right)^{\prime}(w) \leq K \text { for } w \in \Phi_{\eta}^{R}\left(U^{R} \cap(-\mathbb{H})\right), \\
\frac{1}{K} \leq\left(\tau_{+} \circ\left(\Phi_{\eta}^{R}\right)^{-1}\right)^{\prime}(w) \leq K \text { for } w \in \Phi_{\eta}^{R}\left(U^{R} \cap \mathbb{H}\right) .
\end{gathered}
$$

Notice that if $r \leq N$, then all images of $\Delta$ by $\eta$ and its inverse are commensurable by the Koebe Distortion Theorem, hence the statement is evident. Therefore we assume $r>N$. Since $\delta$ is universal, we can restrict ourselves to $z_{0} \in\left(U^{A} \cup U^{R}\right) \cap \eta^{i}(I)$, and $l \leq \delta$. 
By symmetry consider the case $z_{0} \in U^{A}$ only. Given $l \in\left[\left|\eta^{i}(I)\right|, \delta / 2\right]$ one can find real $y \in \eta^{j}(\Delta \cap \mathbb{R})$, such that $\left|y-z_{0}\right| \sim l$ and the circle $U$ of radius $l$ about $y$ lies strictly inside $U^{A}$.

Let us study the intersections of $U_{-}=U \cap(-\mathbb{H})$ with $\tilde{\Delta}$. Firstly, $U_{-}$intersects halfs of $\tilde{\Delta}$, which are the images and preimages of $\Delta$ by $\eta$. However, $\eta$ also has a branching point at 0 , which gives rise to $\tilde{\Delta}$ lying in $-\mathbb{H}$ or $\mathbb{H}$ (see Figure 5 ). In fact, it follows from Lemma 5.2 , that the set $\left\{\Phi_{\eta}^{A}(\tilde{\Delta})\right\}$ is a bounded distortion image of the lattice $\sqcup_{n, m \in \mathbb{Z}} D_{1 / 4}(n+i m$ ) (see Figure 6). By the above estimates, the set $\left\{\tau_{-} \circ\left(\Phi_{\eta}^{A}\right)^{-1} \circ \Phi_{\eta}^{A}(\tilde{\Delta})\right\}=\left\{\tau_{-}(\tilde{\Delta})\right\}$ forms a perturbed lattice in $\tau_{-}\left(U^{A} \cap(-\mathbb{H})\right)$. Consider

$$
\sum_{\tilde{\Delta}} \operatorname{area}\left(\tilde{\Delta} \cap U_{-}\right)=\sum_{\tilde{\Delta}} \iint_{\tau_{-}\left(\tilde{\Delta} \cap U_{-}\right)} J_{\tau_{-}^{-1}}(w) d u d v
$$

where $w=u+i v, d u d v$ is an element of area, $J_{\tau_{-}^{-1}}(w)$ is the Jacobian of $\tau_{-}^{-1}$ at $w$. Since $\tau_{-}^{2}$ is the identity,

$$
\tau_{-}^{-1}(w)=z_{-}-\frac{1}{w-z_{-}}
$$

By the definition of the Jacobian of a conformal map:

$$
J_{\tau_{-}^{-1}}(w)=\left|\left(\tau_{-}^{-1}\right)^{\prime}(w)\right|^{2}=\frac{1}{\left|w-z_{-}\right|^{4}} .
$$

Hence the sum in (6.1) takes the form

$$
\sum_{\tilde{\Delta}} \operatorname{area}\left(\tilde{\Delta} \cap U_{-}\right)=\sum_{\tilde{\Delta}} \iint_{\tau_{-}\left(\tilde{\Delta} \cap U_{-}\right)} \frac{1}{\left|w-z_{-}\right|^{4}} d u d v .
$$

Since $\left\{\tau_{-}(\tilde{\Delta})\right\}$ form a perturbed lattice we can estimate the right-hand side as an integral of $1 /\left|w-z_{-}\right|^{4}$ over $\tau_{-}\left(U_{-}\right)$times some universal constant $C$ :

$$
\sum_{\tilde{\Delta}} \operatorname{area}\left(\tilde{\Delta} \cap U_{-}\right) \geq C \iint_{\tau_{-}\left(U_{-}\right)} \frac{1}{\left|w-z_{-}\right|^{4}} d u d v=C \sigma\left(U_{-}\right)=C \pi l^{2} / 2
$$

(notice that we used here that $y \in \eta^{j}(\Delta \cap \mathbb{R})$, i.e., the intersection of $U_{-}$with $\tilde{\Delta}$ is always non-empty). Similar estimates can be carried over for $U_{+}$, which yields the inequality

$$
\sum_{\tilde{\Delta}} \operatorname{area}(\tilde{\Delta} \cap U) \geq C \pi l^{2} \geq C l^{2}
$$

\section{UNIFORM TWISTING ON THE JULIA SET}

The main result we prove in this section is the following:

Theorem 7.1 (Uniform twisting). There exists a universal constant $C>0$ such that the following statement holds. Let $\zeta$ be a critical commuting pair with an irrational rotation 
number, and let $N$ be such that for all $n>N$ the renormalization $\mathcal{R}^{n} \zeta$ extends to a $K$ bounded holomorphic commuting pair with a universal $K$ (as in (3.4). Fix $n>N$ and let $\mathcal{H}$ be the above extension. Then for every $z_{0} \in J(\mathcal{H})=\cap_{m \geq 0} \mathcal{H}^{-m} \Delta$ and for all $l \in(0,1]$ there exists a pointed area $(U, y)$ such that $\left|z_{0}-y\right| \sim l$, diam $U \sim l$ and

$$
\sum_{\tilde{\Delta} \subset U} \sigma(\tilde{\Delta}) \geq C l^{2},
$$

where $\tilde{\Delta}$ is the range of a holomorphic pair, which is a universally bounded distortion conformal copy of some renormalization of $\mathcal{H}$, generated by the complete dynamics of $\mathcal{H}$.

For $i \geq 0$ set $x_{i}=f^{i}(0)$. Recall the definition of the $m$-th dynamical partition $\mathcal{P}_{m}(2.1)$, and for a point $x \notin\left\{x_{i}, 0 \leq i<q_{m}+q_{m+1}\right\}$ denote $\mathcal{P}_{m}(x)$ the element of the partition containing $x$. For $x=x_{i}$, let $\mathcal{P}_{m}(x)$ be the element of the partition which directly follows $x$ according to the standard choice of the orientation on the circle.

We will find the following two simple lemmas useful in the proof.

Lemma 7.2. Let $f: \mathbb{T} \rightarrow \mathbb{T}$ be a critical circle mapping. Let $J_{m}$ be an open interval with endpoints $f^{q_{m}}(0)$ and $f^{q_{m-1}}(0)$. Then the intervals $J_{m}, f^{-1} J_{m}, \ldots, f^{-q_{m-1}+1} J_{m}$ are all disjoint. Moreover, there exists $M \in \mathbb{N}$ such that for all $m \geq M$ the following holds. Let $\tilde{J}_{m, k}$ be the largest closed interval containing $f^{-k} J_{m}$ such that

$$
\tilde{J}_{m, k} \subset \mathbb{T} \backslash \underset{j \in\left\{0, \ldots, q_{m-1}-1\right\} \backslash\{k\}}{\bigcup} f^{-j} J_{m} .
$$

Then $f^{-k} J_{m}$ lies universally well-inside $\tilde{J}_{m, k}$ and $\left|f^{-k} J_{m}\right| \geq\left|\tilde{J}_{m, k}\right| / C$ for some universal $C>1$.

Proof. Let us recall that

$$
\mathcal{P}_{m-2}=\left\{\begin{array}{ll}
I_{m-1}^{j}, & 0 \leq j<q_{m-2} \\
I_{m-2}^{j}, & 0 \leq j<q_{m-1}
\end{array}\right\} \text { and } \mathcal{P}_{m-1}=\left\{\begin{array}{ll}
I_{m}^{j}, & 0 \leq j<q_{m-1} \\
I_{m-1}^{j}, & 0 \leq j<q_{m}
\end{array}\right\}
$$

Notice that $J_{m} \subset I_{m-1} \cup I_{m-2}$ and $f^{-q_{m-1}} J_{m} \subset I_{m-2}$ : Therefore

$$
\begin{aligned}
f^{-1} J_{m} & \subset I_{m-2}^{q_{m-1}-1}, \\
\vdots & \\
f^{-i} J_{m} & \subset I_{m-2}^{q_{m-1}-i}, \\
\vdots & \\
f^{-q_{m-1}+1} J_{m} & \subset I_{m-2}^{q_{m-1}-q_{m-1}+1}=I_{m-2}^{1} .
\end{aligned}
$$

Hence the intervals $J_{m}, f^{-1} J_{m}, \ldots, f^{-q_{m-1}+1} J_{m}$ are disjoint. A priori bounds for $\mathcal{P}_{m-2}$ imply that the adjacent intervals $I^{\prime}, I^{\prime \prime} \in \mathcal{P}_{m-2}$ are commensurable. A priori bounds also 
imply that

$$
\frac{1}{C_{0}}\left|I_{m-2}^{q_{m-1}-i}\right| \leq\left|f^{-i} J_{m}\right| \leq C_{0}\left|I_{m-2}^{q_{m-1}-i}\right|
$$

The partition $\mathcal{P}_{m-2}$ may contain at most one interval of the orbit $I_{m-1}^{j}$ between the interval $I_{m-2}^{q_{m-1}-k} \supset f^{-k} J_{m}$ and the interval of the orbit $I_{m-2}^{j}$ which follows it. Therefore the required bound for $\tilde{J}_{m, k}$ holds true.

Lemma 7.3. Let $\mathcal{H}=(\xi, \eta)$ be a universally bounded holomorphic pair. Then there exists a disk $D^{\prime} \subset D \backslash \mathbb{R}$ and a disk $D^{\prime \prime} \subset \Omega_{\mathcal{H}}$, centered at the origin, with diam $D^{\prime} \sim|J| \sim \operatorname{diam} D^{\prime \prime}$ and $\operatorname{dist}\left(D^{\prime}, \mathbb{R}\right) \sim J$, such that $\nu=\xi \circ \eta$ is univalent in $D^{\prime}$ and $D^{\prime \prime} \subset \nu\left(D^{\prime}\right)$.

Proof. Follows from the fact that $\nu$ is cubic up to a universally bounded distortion.

The first step towards proving Theorem 7.1 is:

Lemma 7.4. There exists $C>0$ independent of $\zeta$ such that for all $n>N$ as in the statement of Theorem 7.1 the following statement holds. Let $\mathcal{H}$ be a K-bounded holomorphic pair extension of $\mathcal{R}^{n} \zeta$. For any $z_{0} \in[\xi(0), \eta(0)]$ and for any $l \in(0,1]$ there exists a pointed area $(U, y)$ such that $\left|z_{0}-y\right| \sim l$, $\operatorname{diam} U \sim l$ and the estimate (7.1) holds.

Proof. Let $f=\left.\mathcal{H}\right|_{[\xi(0), \eta(0)]}, \rho(f)=\left[r_{0}, r_{1}, \ldots\right], p_{m} / q_{m}=\left[r_{0}, \ldots, r_{m-1}\right]$ and for each $m$ choose $J_{m}$ as in Lemma [7.2. Thus $J_{m}$ is the dynamical interval of the $m$-th pre-renormalization $\mathcal{H}_{m}$ of $\mathcal{H}$. Let $\Delta_{m} \supset J_{m}$ be the range of $\mathcal{H}_{m}$. Complex bounds (Theorem 3.8) imply that $\operatorname{diam} \Delta_{m} \sim\left|J_{m}\right|$. For each $0<i \leq q_{m-1}-1$, let $V_{m}^{(i)}=f^{-i} \Delta_{m}$. By the Koebe Distortion Theorem we have diam $V_{m}^{(i)} \sim\left|f^{-i} J_{m}\right|$. Now let us choose minimal $m$ such that

$$
\left|\mathcal{P}_{m}\left(z_{0}\right)\right| \leq l \leq\left|\mathcal{P}_{m-1}\left(z_{0}\right)\right|
$$

Consider $r_{m}=\chi\left(\mathcal{H}_{m}\right)$, recalling that $q_{m+1}=r_{m} q_{m}+q_{m-1}$. Consider $m>M$ and take $N$ as in the proof of the Lemma 6.1] If $r_{m} \leq N$, then

$$
\frac{\left|\mathcal{P}_{m}\left(z_{0}\right)\right|}{\left|\mathcal{P}_{m-1}\left(z_{0}\right)\right|} \geq \frac{1}{C(N)},
$$

uniformly in $z_{0}$, where $C(N)>1$ is chosen by real a priori bounds and satisfies the additional condition $1 / C(N)<K_{1}$, where $K_{1}>0$ is some universal number to be determined in the course of the proof. Hence for $r_{m} \leq N$ we can simply choose $k$ so that $f^{-k} J_{m}$ is the closest to $z_{0}$. By Lemma 7.2 and commensurability of $f^{-k} J_{m}$ and $V_{m}^{(k)}$, this value of $k$ and the domain $(U, y)=\left(V_{m}^{(k)}, f^{-k}(0)\right)$ satisfy the conditions of the Lemma.

Let us now consider the other case, when $r_{m}>N$ and the ratio

$$
\left|\mathcal{P}_{m}\left(z_{0}\right)\right| /\left|\mathcal{P}_{m-1}\left(z_{0}\right)\right|
$$

is smaller when $1 / C(N)$. The only complicated case with "possible parabolic cascade" in this situation is

(i) $\mathcal{P}_{m-1}\left(z_{0}\right)=I_{m-1}^{t}$ for some $0 \leq t<q_{m}$;

(ii) $\mathcal{P}_{m}\left(z_{0}\right)=I_{m}^{s}$ for some $0 \leq s<q_{m+1}$; 
Indeed, it can not happen that $\mathcal{P}_{m-1}\left(z_{0}\right)=I_{m}^{j}, 0 \leq j<q_{m-1}$, since then $\mathcal{P}_{m}\left(z_{0}\right)=I_{m}^{j}$, and $\left|\mathcal{P}_{m}\left(z_{0}\right)\right| /\left|\mathcal{P}_{m-1}\left(z_{0}\right)\right|=1$ and we can choose $k$ so that $V_{m}^{(k)}$ is the closest to $z_{0}$ as in previous paragraphs.

If $\mathcal{P}_{m}\left(z_{0}\right)=I_{m+1}^{s}, 0 \leq s<q_{m}$, then $I_{m+1}^{s} \subset I_{m-1}^{s}=\mathcal{P}_{m-1}\left(z_{0}\right)$, i.e., $s=t$ and a priori estimates imply that they are comparable, i.e., $\left|\mathcal{P}_{m}\left(z_{0}\right)\right| /\left|\mathcal{P}_{m-1}\left(z_{0}\right)\right|>K_{1}$, where $K_{1}$ is a constant whose existence was announced above. Similarly, we can choose $k$ so that $V_{m}^{(k)}$ is the closest to $z_{0}$, and domain $(U, y)=\left(V_{m}^{(k)}, f^{-k}(0)\right)$ works.

Let us sketch the position of intervals in the case of "possible parabolic cascade":

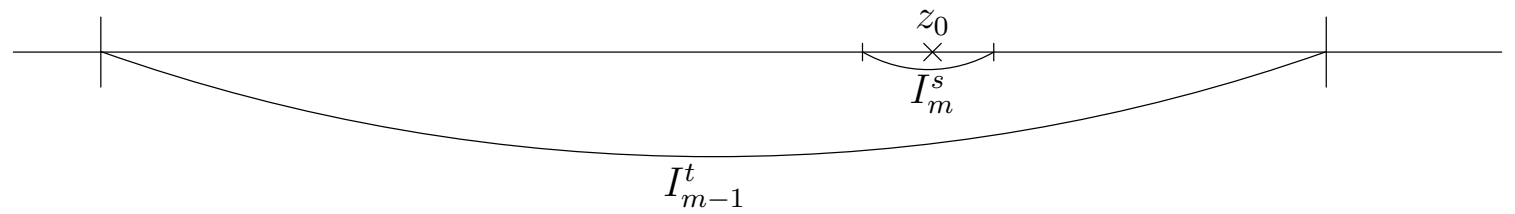

Applying $f^{-t}$ to $I_{m-1}^{t}$ we arrive exactly into situation described in Lemma 6.1.

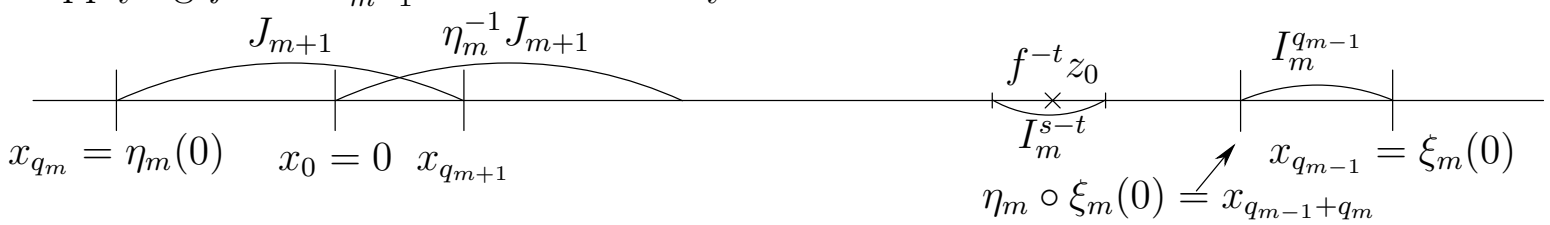

Notice that $I_{m}^{s-t} \neq I_{m}^{q_{m-1}}$ (otherwise $\left.\left|\mathcal{P}_{m}\left(z_{0}\right)\right| /\left|\mathcal{P}_{m-1}\left(z_{0}\right)\right|>K_{1}\right)$ and $I_{m}^{s-t} \cap J_{m+1}=\varnothing$ (by combinatorial reasons).

Let $\left(\eta_{m}, \xi_{m}\right)=\mathcal{R}^{m}(\mathcal{H})$. Consider $J_{m+1}$ and corresponding range $\Delta=\Delta_{m+1}$. Obviously, $\eta_{m}^{-1} J_{m+1} \subset I_{m-1}$ and $\eta_{m}^{-2} J_{m+1}$ lies well-inside $I_{m-1}$. The same branch $\eta_{m}^{-1}$ pullbacks $\eta_{m}^{-1} \Delta$ (if $\eta_{m}^{-1} \Delta \ni 0$, we can consider $\Delta=\Delta_{m+k}$, such that $\eta_{m}^{-1} \Delta \not \supset 0$, where $k$ is universally bounded). By Lemma 6.1 we can find a pointed area $\left(U^{\prime}, y^{\prime}\right),\left|y^{\prime}-f^{-t} z_{0}\right| \sim l$, diam $U^{\prime} \sim l$, which intersects a large number of images of $\eta_{m}^{-1} \Delta$ under the complete dynamics of $\eta_{m}$. Moreover, $\operatorname{dist}\left(U^{\prime}, 0\right) \sim\left|I_{m-1}\right|$. Hence an application of the Koebe Distortion Theorem gives $(U, y)=\left(f^{t}\left(U^{\prime}\right), f^{t} y^{\prime}\right)$ which satisfies the conditions of the theorem.

By passing to renormalizations of $\zeta$ one can easily derive the following version of the above lemma:

Proposition 7.5. There exists $C$ such that for all $n$ large enough the following holds. Let $\mathcal{H}: \Omega \rightarrow \Delta$ be a K-bounded holomorphic pair extension of $\mathcal{R}^{n} \zeta$. Then for every $z_{0} \in \Omega \cap \mathbb{R}$ and for all $l \in(0,1]$ there exists a pointed area $(U, y)$ such that $\left|z_{0}-y\right| \sim l$, diam $U \sim l$ and (7.1) holds.

Proof of Theorem 7.1. The proof is similar to steps II and III of the proof of dFdM1, Theorem 6.8] with two essential differences. The first difference is that we have to take into account possible parabolic cascades and to use Lemma 6.1 to work with them. The second difference is that we should treat specially the cases when $\chi(\mathcal{H})$ is large and the trajectory $z_{k}=\mathcal{H}^{k} z$ approaches the real axis along the wings of the "butterfly", not near 


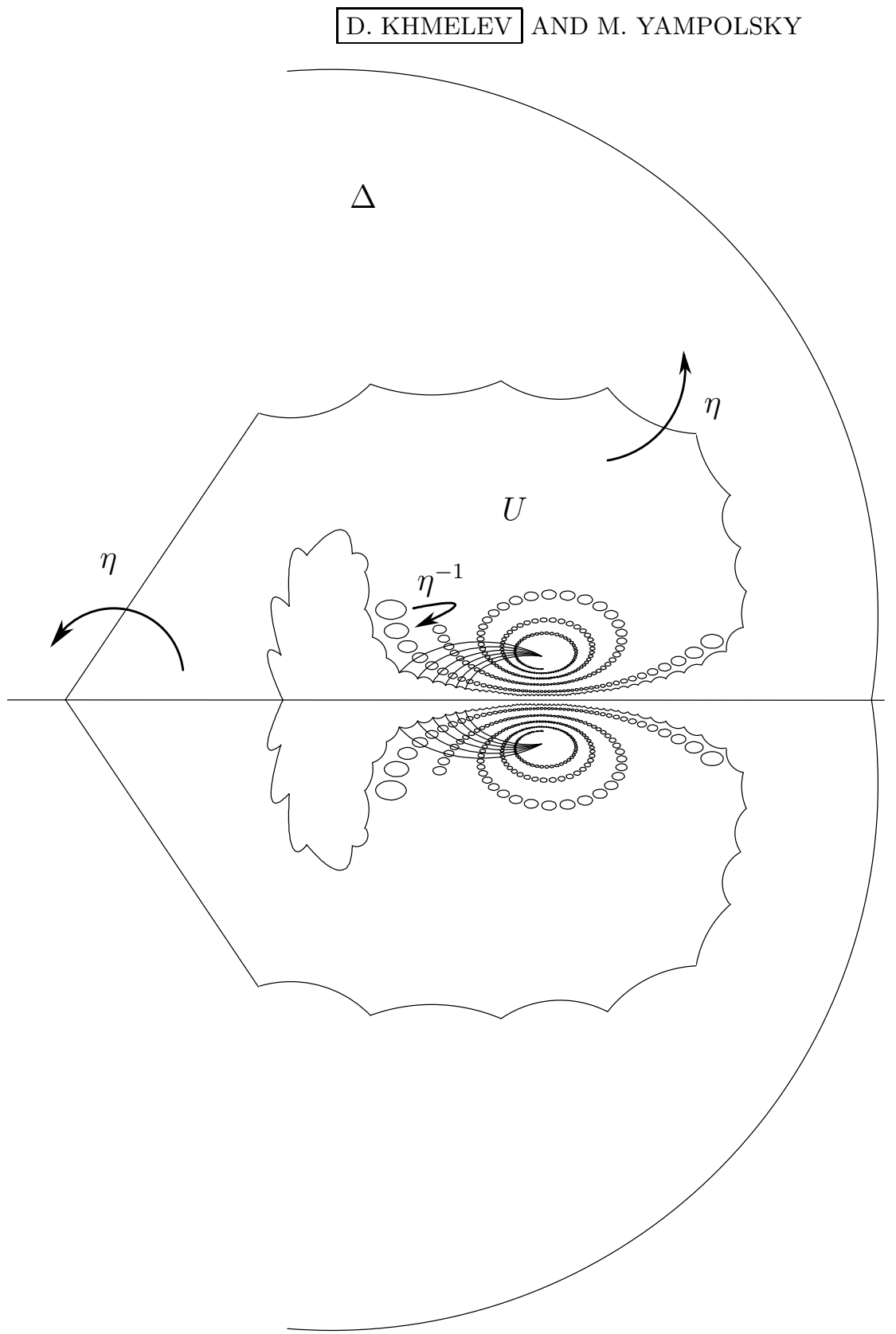

FiguRE 7. Butterfly wing with parabolic behaviour $(\chi(\mathcal{H})>N)$

the interval $\Omega_{\mathcal{H}} \cap \mathbb{R}$ (see Figure [3); we use Lemma 6.1] together with Lemma 3.10 to get the estimates in this case.

From now on assume $z_{0} \in J(\mathcal{H}) \backslash \mathbb{R}$. By symmetry it is enough to consider the case $z_{0} \in \mathbb{H} \cap J(\mathcal{H})$. Let $H=\Delta \backslash \mathbb{R}$ and consider a vector $v_{0}$ at $z_{0}$ with the Euclidian norm $\left|v_{0}\right| \sim l$. The sketch of the argument, which goes back to McMullen, is as follows: consider the iterations $z_{k}=\mathcal{H}^{k} z_{0}, v_{k}=\left(\mathcal{H}^{k}\left(z_{0}\right)\right)^{\prime} v_{0}$ and wait until the disk of radius $v_{k}$ around $z_{k}$ 
encloses a commesurable $\left(U^{\prime}, y^{\prime}\right)$ disk with desired property (17.1). Afterwards we can pull $\left(U^{\prime}, y^{\prime}\right)$ back to $z_{0}$ to get the required $(U, y)$. Notice that $z_{k}$ never leaves $J(\mathcal{H})$, while the hyperbolic length $\ell_{H}\left(v_{k}\right)$ of $v_{k}$ increases to infinity (see Theorem 4.11, dFdM2 ):

$$
\ell_{H}\left(v_{0}\right) \leq \ell_{H}\left(v_{1}\right) \leq \ldots \leq \ell_{H}\left(v_{k}\right) \rightarrow \infty
$$

There are two cases to consider.

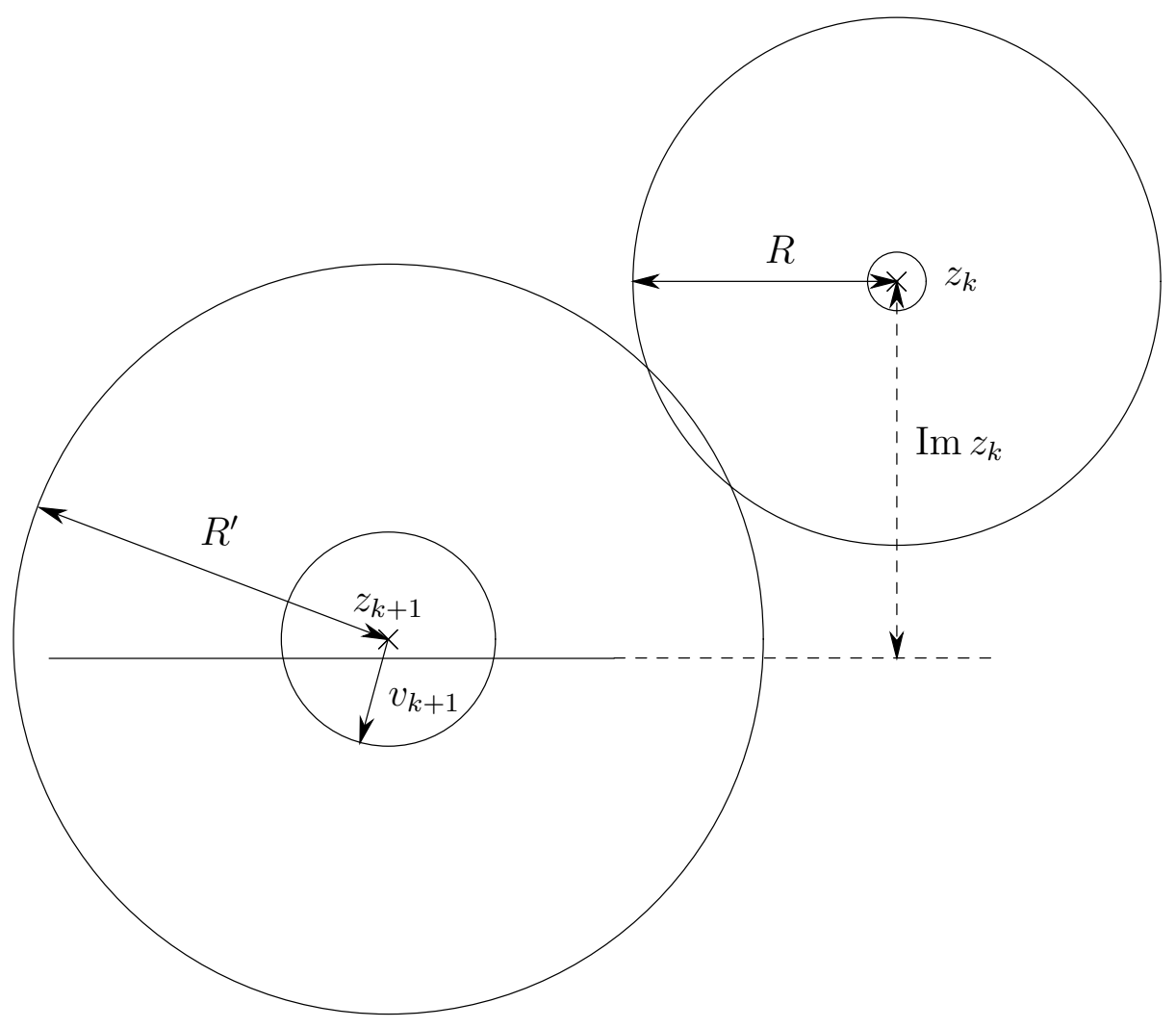

Figure 8. Case of $\ell_{H}\left(v_{k}\right)<\epsilon$ and $\ell_{H}\left(v_{k+1}\right)>1 / \epsilon$

Case $A$. There exists $k$ such that $\ell_{H}\left(v_{k}\right)<\epsilon$ while $\ell_{H}\left(v_{k+1}\right)>1 / \epsilon$, where $\epsilon$ is a universal constant to be determined in the course of the argument. To be definite, assume $z_{k}$ lies in the domain of $\eta$, so $z_{k+1}=\eta\left(z_{k}\right)$. Since for all $z$ in domain $\Omega$ of $\mathcal{H}$, the hyperbolic density of $\mathcal{H}$ is commensurable with $1 / \operatorname{Im} z$, we have $\left|v_{k}\right| / \operatorname{Im} z_{k} \sim \ell_{H}\left(v_{k}\right)<\epsilon$. Hence $\operatorname{Im} z_{k} \geq C\left|v_{k}\right| / \epsilon>>\left|v_{k}\right|$. Since all the branching points belong to the real axis, $\eta$ is univalent in a disk $D_{R}\left(z_{k}\right)$ of radius $R \sim \operatorname{Im} z_{k}$ (notice, that we may need to consider the renormalization of $\mathcal{H}$ instead of $\mathcal{H}$ to be able to extend $\eta$ holomorphically to the disk), cf Figure 8. By the Koebe One-quarter Theorem, $\eta\left(D_{R}\left(z_{k}\right)\right)$ contains the disk $D_{R^{\prime}}\left(z_{k+1}\right)$, where $R^{\prime}=R\left|v_{k+1}\right| / 4\left|v_{k}\right|>>\left|v_{k+1}\right|$. But $\ell_{H}\left(v_{k+1}\right) \sim\left|v_{k+1}\right| / \operatorname{Im} z_{k+1}$ is large $(>1 / \epsilon)$, hence $\left|v_{k+1}\right|>>\operatorname{Im} z_{k+1}$. Now consider two subcases. 
Subcase $A^{\prime}$. Assume that $\chi(\mathcal{H}) \leq N, N$ being a universal number to be fixed later. Then the distance of the wings from the real axis is greater than some constant $\epsilon^{\prime}=\epsilon^{\prime}(N)>0$. Hence, there exists constant $\epsilon_{1}=\epsilon_{1}(N)$, such that for any $0<\epsilon<\epsilon_{1}$ we can take the point $\zeta$ in the interval $\Omega \cap \mathbb{R}$, closest to $z_{k+1}$, and $\operatorname{dist}\left(\zeta, z_{k+1}\right) \sim \operatorname{Im} z_{k+1}$. By Proposition 7.5 there exists a pointed domain $\left(U^{\prime \prime}, y^{\prime \prime}\right)$ with the property (7.1) , such that diam $U^{\prime \prime} \sim\left|v_{k+1}\right|$ and $\left|\zeta-y^{\prime \prime}\right| \sim\left|v_{k+1}\right|$, and therefore $\left|z_{k+1}-y^{\prime \prime}\right| \sim\left|v_{k+1}\right|$. If $\epsilon<\epsilon_{1}$ is chosen small enough, we have $U^{\prime \prime} \subset D_{R^{\prime} / 2}\left(z_{k+1}\right)$. Take $U^{\prime}=\eta^{-1}\left(U^{\prime \prime}\right) \subset D_{R}\left(z_{k}\right)$ and $y^{\prime}=\eta^{-1} y^{\prime \prime} \in U^{\prime}$. By the Koebe Distortion Theorem $\operatorname{diam}\left(U^{\prime}\right) \sim\left|v_{k}\right|$, and $\left|z-v_{k}\right| \sim\left|v_{k}\right|$. Since $D_{R}\left(z_{k}\right) \subset \mathbb{H}$, we can pull back $\left(U^{\prime}, y^{\prime}\right)$ using the univalent inverse branch of $\mathcal{H}^{-k}$ mapping $z_{k}$ to $z_{0}$ to get $(U, y)=\left(\mathcal{H}^{-k} U^{\prime}, \mathcal{H}^{-k} y^{\prime}\right)$, which by the Koebe Theorem satisfies (17.1) with a finitely distorted constant $C$.

Subcase $A^{\prime \prime} . \chi(\mathcal{H})>N$. This case is illustrated by Figure 7 Lemma 3.10 guarantees that the mapping $\eta$ is close to a parabolic one in a disk $\hat{D}$, which is commensurable with $I_{\eta}$. At the same time since $\eta$ also has a branching point at the endpoint of the interval $\Omega \cap \mathbb{R}$, we can bring a copy of holomorphic pair, say $\mathcal{R H}$, commesurable with the length of $\gamma_{m}^{i}$ inside $\hat{D}$ and use the method of the proof of Lemma 6.1 to spread it around taking images and pre-images with $\eta$. Obviously, if $z_{k+1}$ is outside $\hat{D}$ it can be treated as in Subcase $A^{\prime}$.

Assume that $z_{k+1}$ is inside $\hat{D} \cap U$. In order to apply estimates similar to the one used in the proof of Proposition 7.5 we have to guarantee that for $\tilde{\Delta}$ closest to $z_{k+1}$ the diam $\tilde{\Delta}$ is smaller than some constant times $\left|v_{k+1}\right|$.

Recall that $\left|v_{k+1}\right|>>\operatorname{Im} z_{k+1}$, hence we fall into one of the situations, sketched on Figure 9. In "nondegenerate case" $z_{k+1}$ lies inside the lattice of disks, which together with Lemma 6.1 implies that disk $D_{R^{\prime}}\left(z_{k+1}\right)$ encloses pointed region $\left(U^{\prime \prime}, y^{\prime \prime}\right)$ with the property (7.1) and further argument is the same as in Subcase $A^{\prime}$.

The degenerate situation, sketched in Figure 9 has, in fact, bounded geometry. Indeed, if $z_{k+1}$ lies outside the lattice of disks, consider disk $\tilde{\Delta}$ and a piece of boundary $\gamma_{m}^{i}$, both closest to $z_{k+1}$ (cf Figure [7). From estimates, given in Ya1, and boundedness of derivatives of $\mathcal{E}_{\eta}^{-1}$ we obtain $\operatorname{dist}\left(\gamma_{m}^{i}, \mathbb{R}\right) \sim \operatorname{dist}\left(\gamma_{m}^{i}, \tilde{\Delta}\right) \sim \operatorname{diam} \tilde{\Delta}$ universally. Hence $\operatorname{Im} z_{k+1} \geq K_{0} \operatorname{dist}\left(\gamma_{m}^{i}, \mathbb{R}\right)$, and hence $\left|v_{k+1}\right| \geq K_{0} \operatorname{Im} z_{k+1}$ and one can take $\left(\tilde{\Delta}, y^{\prime \prime}\right)$, $y^{\prime \prime} \in \Delta$, as a pointed domain $\left(U^{\prime \prime}, y^{\prime \prime}\right)$ with property (7.1), such that $\operatorname{diam} U^{\prime \prime} \sim\left|v_{k+1}\right|$ and $\left|z_{k+1}-y^{\prime \prime}\right| \sim\left|v_{k+1}\right|$. Now the same procedure as in Subcase $A^{\prime}$ can be followed (possibly with a further correction of $\epsilon$ ).

Case $B . \ell_{H}\left(v_{k}\right) \sim 1$ for some $k$. Remember that $\operatorname{Im} z_{k+1} \sim\left|v_{k}\right|$.

Subcase $B^{\prime} . \quad \chi(\mathcal{H})>N$ and $z_{k} \in \hat{D}$. If $z_{k}$ is above the repelling fixed point $z_{+}$(i.e. $\operatorname{Im} z_{k} \geq \operatorname{Im} z_{+}$), then by estimates similar to the one used in the proof of Lemma [7.4 there exists a domain $\left(U^{\prime}, y^{\prime}\right)$ with property (7.1) such that diam $U^{\prime} \sim\left|v_{k+1}\right|$ and $\left|z_{k+1}-y^{\prime}\right| \sim$ $\left|v_{k+1}\right|$. This $\left(U^{\prime}, y^{\prime}\right)$ is now easily pulled back by $\mathcal{H}^{-k}$. If $z_{k}$ is below the fixed point $z_{+}$, then there are two subcases depending on the $l=\operatorname{dist}\left(z_{k}, \gamma_{m}^{i}\right)$, where $\gamma_{m}^{i}$ is the closest piece of $\hat{D} \cap \Omega$ to $z_{k}$.

These subcases are similar to the "non-degenerate" and "degenerate" situations, considered in Subcase $A$ " with only the difference that in the "degenerate" situation one can 


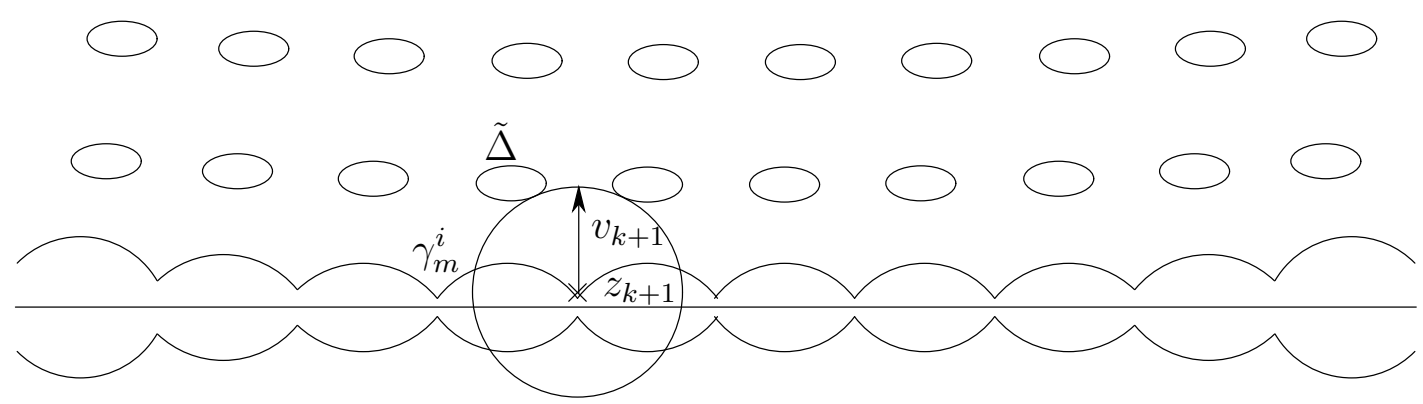

"Degenerate case"

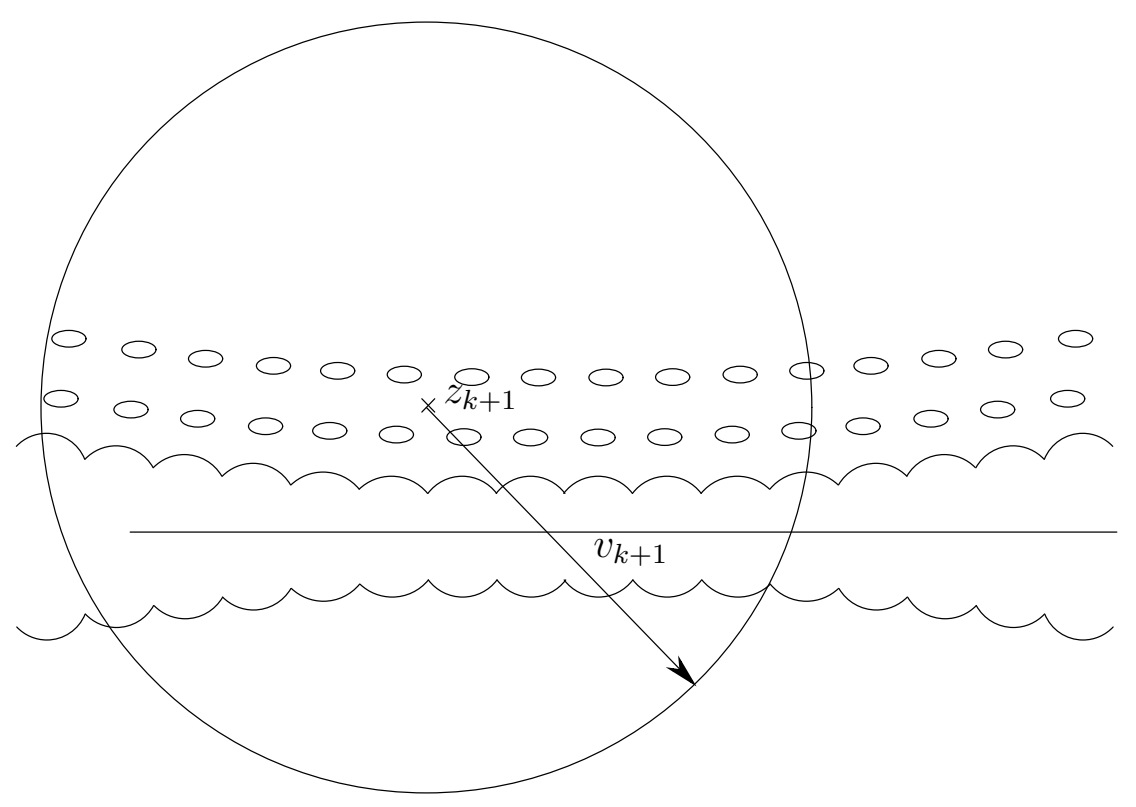

"Non-degenerate case"

FiguRE 9. $z_{k+1}$ and parabolic cascade in wings

not hope for a large disk $D_{R^{\prime}}\left(z_{k+1}\right)$, enclosing many disks from the parabolic lattice (because $R^{\prime}$ is comparable with $\left|v_{k+1}\right|$ here). Hence, in the "degenerate" situation we iterate $z_{k+1}$ further, until such a disk would emerge. The exact description of dynamics, given by Lemma 3.10 allows us to do so. Next, we describe this procedure more formally.

If $l>K_{1}\left|v_{k}\right|$ for some $K_{1}$, then we can use the same estimates as in Lemma 7.4 and the argument is similar to the one used in subcase $A^{\prime \prime}$. Otherwise $z_{k}$ becomes quite close to $\gamma_{m}^{i}$ and further application of $\eta$ moves $z_{k}$ along the parabolic cascade, increasing the hyperbolic length of $\ell_{H}\left(v_{k}\right)$ (see Lemma 3.10 for description of dynamics). 
Eventually, we either fall into Subcase $A^{\prime \prime}$ (with the large hyperbolic length and the possible pull-back of the large number of copies of $\tilde{\Delta})$, or $\ell_{H}\left(v_{l}\right)$ remains comparable with 1 (which also allows to pull back a disk with copies of $\tilde{\Delta}$ at the moment $l$ when $z_{l} \in \hat{D}$, $\left.z_{l+1} \notin \hat{D}\right)$.

Subcase $B^{\prime \prime}$. Situations $(1) \chi(\mathcal{H}) \leq N$ and $(2) \chi(\mathcal{H})>N$ and $z_{k} \notin \hat{D}$ can be considered simultaneously.

Let $\zeta$ be the point in $\Omega \cap \mathbb{R}$, closest to $z_{k}$. Then by Proposition [7.5 there exists a pointed domain $\left(U^{\prime \prime}, y^{\prime \prime}\right), y^{\prime \prime} \in \mathbb{R}$, such that $\left|\zeta-y^{\prime \prime}\right| \sim \operatorname{Im} z_{k}$, $\operatorname{diam} U^{\prime \prime} \sim \operatorname{Im} z_{k}$, satisfying the condition (17.1). It follows from the proof of Lemma 7.4 that one of the following conditions holds for some universal $K_{2}$ :

i) There exists $\tilde{\Delta} \subset U^{\prime \prime}$ such that $\operatorname{diam} \tilde{\Delta} \geq K_{2} \operatorname{diam} U^{\prime \prime}, \tilde{\Delta} \cap \mathbb{R} \neq \varnothing$.

ii) There exists a disk $U^{\prime}$ around $y^{\prime}, U^{\prime} \subset U^{\prime \prime}$, with the property (7.1), such that $\operatorname{diam} U^{\prime} \geq K_{2} \operatorname{diam} U^{\prime \prime}$ and $\operatorname{dist}\left(U^{\prime}, \mathbb{R}\right) \sim \operatorname{diam} U^{\prime \prime}$.

In case i) we can pull-back $\tilde{\Delta}$ to origin by $\mathcal{H}^{-k}$ for some $k$ and to produce a disk $D^{\prime}$, containing a preimage of a holomorphic pair, commensurable with $D^{\prime}$, using Lemma 7.3 , Now we choose $\left(U^{\prime}, y\right)=\left(\mathcal{H}^{k} D^{\prime}, \mathcal{H}^{k} d\right)$, where $d$ is the center of $D^{\prime}$. Notice that by the Koebe Theorem $U^{\prime}$ has the property (17.1).

In case ii) we already have $U^{\prime}$ satisfying (7.1).

The remaining step is to pull-back $\left(U^{\prime}, y^{\prime}\right)$ to $z_{0}$, which can be carried over as in dFdM1, Theorem 6.8, step IIIb]. Indeed, if $\gamma$ is a geodesic arc in $H$, connecting $z_{k}$ to $U^{\prime}$, then $\operatorname{diam}\left(\gamma \cup U^{\prime}\right) \sim \operatorname{Im} z_{k}$. Hence there exist $r_{k} \sim \operatorname{Im} z_{k}$ and $\zeta_{1}=z_{k}, \zeta_{2}, \ldots, z_{s} \in \gamma \cup U^{\prime}$, such that

$$
\gamma \cup U \subset \mathcal{V}=\cup_{j=1}^{s} D_{r_{k} / 2}\left(\zeta_{j}\right)
$$

and such that $D_{r_{k}}\left(\zeta_{j}\right) \subset \Delta \backslash \mathbb{R}$ for all $j$. Inverse branch $\Psi=\mathcal{H}^{-k}$, taking $z_{k}$ to $z_{0}$ is defined in each $D_{r_{k}}\left(\zeta_{j}\right)$. Application of the Koebe Theorem to each $D_{r_{k}}\left(\zeta_{j}\right)$ yields $\left|\Psi^{\prime}(z)\right| \sim\left|\Psi^{\prime}\left(z_{k}\right)\right| \sim$ $\left|v_{0}\right| /\left|v_{k}\right|$. Hence $(U, y)=\left(\Psi\left(U^{\prime}\right), \Psi\left(y^{\prime}\right)\right)$ satisfy $\operatorname{diam} U \sim\left|v_{0}\right|, \operatorname{dist}\left(y, z_{0}\right) \sim\left|v_{0}\right|$.

Proof of Theorem 4.5. In view of Proposition 4.4, the abundance of copies of holomorphic commuting pairs given by the estimate (7.1) provides a uniform and universal bound from below on nonlinearity defined by (4.1).

Proof of Theorem 1.1. The statement follows from the Dynamical Inflexibility Theorem 4.1 together with Theorem 4.2 (I) and Theorem 4.5.

\section{Hyperbolicity of the RENORMALIZATION HORSESHOE}

In this section we will apply our Rigidity Theorem to obtain a new proof of the hyperbolicity of the global horseshoe for the cylinder renormalization operator $\mathcal{R}_{\text {cyl }}$ Ya3, Ya4. The second author has established this result in [Ya4 using some infinite-dimensional quasiconformal deformation spaces arguments. Now we will be able to give a simpler argument, following along the same lines as the proof of hyperbolicity of the periodic orbits of $\mathcal{R}_{\mathrm{c} y l}$ given in Ya3. We will use Ya3 as the general reference for this section, however, for the sake completeness, we will briefly recall the basic definitions. 
Definition of $\mathcal{R}_{\mathbf{c} y \mathbf{l}}$. The main point of Ya3 was to replace the renormalization operator $\mathcal{R}$ acting on the space of commuting pairs with an analytic operator $\mathcal{R}_{\text {cyl }}$ defined on a complex-analytic Banach manifold. To define $\mathcal{R}_{\text {cyl }}$, we will need a few preliminaries. Firstly, let us denote $\pi: \mathbb{C} \rightarrow \mathbb{C} / \mathbb{Z}$ the natural projection. For an equatorial topological annulus $U \subset \mathbb{C} / \mathbb{Z}$ denote $\mathbf{A}_{U}$ the space of bounded analytic maps $\varphi: U \rightarrow \mathbb{C} / \mathbb{Z}$, such that $\varphi(\mathbb{T})$ is homotopic to $\mathbb{T}$, equipped with the uniform metric. To turn this space into a Banach manifold, consider the Banach space $\tilde{\mathbf{A}}_{U}$ of bounded analytic 1-periodic functions from $\pi^{-1}(U) \rightarrow \mathbb{C}$ with the sup norm, and use the local homeomorphism $\tilde{\mathbf{A}}_{U} \rightarrow \mathbf{A}_{U}$ given by

$$
\psi \mapsto \pi \circ(\psi+\mathrm{Id}) \circ \pi^{-1}
$$

to define the atlas on $\mathbf{A}_{U}$. We denote $\mathbf{C}_{U}$ the codimension two submanifold of $\mathbf{A}_{U}$ consisting of maps with a cubic critical point at the origin. Let $\mathbf{C}_{U}^{\mathbb{R}} \subset \mathbf{C}_{U}$ be the real Banach manifold consisting of the critical circle maps in $\mathbf{C}_{U}$ (the real slice of $\mathbf{C}_{U}$ ). As in Ya3, a tangent space to $\mathbf{C}_{U}$ will be naturally identified with a Banach subspace $\mathbf{B}_{U} \subset \tilde{\mathbf{A}}_{U}, \mathbf{B}_{U}^{\mathbb{R}}$ will again denote the real slice.

Given a critical cylinder map $f \in \mathbf{C}_{U}$ let us say that it is cylinder renormalizable, or simply renormalizable, if there exists $k>1$ and an equatorial annulus $V \subset \mathbb{C} / \mathbb{Z}$ such that following holds:

- there exist repelling periodic points $p_{1}, p_{2}$ of $f$ in $U$ with periods $k$ and a simple arc $l$ connecting them such that $f^{k}(l)$ is a simple arc, and $f^{k}(l) \cap l=\left\{p_{1}, p_{2}\right\}$;

- the iterate $f^{k}$ is defined and univalent in the domain $C_{f}$ bounded by $l$ and $f^{k}(l)$, the corresponding inverse branch $\left.f^{-k}\right|_{f^{k}\left(C_{f}\right)}$ univalently extends to $C_{f}$; and the quotient of $\overline{C_{f} \cup f^{k}\left(C_{f}\right)} \backslash\left\{p_{1}, p_{2}\right\}$ by the action of $f^{k}$ is a Riemann surface conformally isomorphic to the cylinder $\mathbb{C} / \mathbb{Z}$ (we will call a domain $C_{f}$ with these properties a fundamental crescent of $\left.f^{k}\right)$;

- for a point $z \in \bar{C}_{f}$ with $\left\{f^{j}(z)\right\}_{j \in \mathbb{N}} \cap \bar{C}_{f} \neq \emptyset$, set $R_{C_{f}}(z)=f^{n(z)}(z)$ where $n(z) \in \mathbb{N}$ is the smallest value for which $f^{n(z)}(z) \in \bar{C}_{f}$. We further require that there exists a point $c$ in the domain of $R_{C_{f}}$ such that $f^{m}(c)=0$ for some $m<n(c)$; and if we denote $\hat{f}$ the projection of $R_{C_{f}}$ to $\mathbb{C} / \mathbb{Z}$ with $c \mapsto 0$, then $\hat{f} \in \mathbf{C}_{V}$.

We will say that the new critical circle map $\hat{f}$ is a cylinder renormalization of $f$ with period $k$.

Proposition 8.1 (Properties of cylinder renormalization, Ya3]). The following statements hold:

- The cylinder renormalization does not depend on the choice of the fundamental crescent.

- If $f \in \mathbf{C}_{U}$ is cylinder renormalizable with period $k$, then there exists an open neighborhood $\mathcal{W}(f) \subset \mathbf{C}_{U}$ such that every $g \in \mathcal{W}(f)$ is also cylinder renormalizable, with the same period, and the fundamental crescent $C_{g}$ can be chosen to move continuously with $g$. 
- Moreover, the cylinder renormalization is an analytic operator $\mathcal{W}(f) \rightarrow \mathbf{C}_{V}$.

The connection with the renormalization of commuting pairs is established as follows:

Proposition 8.2 (Cylinders of commuting pairs in $\mathcal{E}$ Ya3]). Let $\zeta=(\eta$, $\xi$ ) be a commuting pair in the Epstein class with $\chi(\zeta) \neq \infty$. Then the map $\eta$ has a real-symmetric fundamental crescent $C_{\eta}$, with

$$
C_{\eta} \cup \overline{\eta\left(C_{\eta}\right)} / \eta \simeq \mathbb{C} / \mathbb{Z}
$$

whose first return map projects to an analytic critical circle map $f_{\zeta}$ independent of the choice of $C_{\eta}$, whose rotation number $\rho\left(f_{\zeta}\right)=\rho(\mathcal{R} \zeta)$. Further, there exists $U=U(s)$ such that if $\zeta \in \mathcal{E}_{s}$, then $f_{\zeta} \in \mathbf{C}_{U}^{\mathbb{R}}$.

Proposition 8.3 ([Ya3] $)$. If $\zeta_{1}, \zeta_{2} \in \mathcal{E}$, and $\chi\left(\zeta_{1}\right)=\chi\left(\zeta_{2}\right)=r \neq \infty$, then the equality

$$
f_{\zeta_{1}} \equiv f_{\zeta_{2}}
$$

is equivalent to the existence of a conformal conjugacy between $\zeta_{1}$ and $\zeta_{2}$ whose domain contains a fundamental crescent (we will write $\zeta_{1} \widetilde{\text { conf }}_{\zeta_{2}}$ in this case). Moreover, if $\zeta_{1}, \zeta_{2} \in$ $\mathcal{E}_{s}$, then

$$
\operatorname{dist}_{C^{0}}\left(\zeta_{1}, \zeta_{2}\right) \geq c(s, r) \operatorname{dist}_{C^{0}}\left(f_{\zeta_{1}}, f_{\zeta_{2}}\right) .
$$

From now on, let us fix $s>0$ as in Lemma 3.3, and set $U=U(s)$ from Proposition 8.2 , Finally, we have:

Proposition 8.4. Then there exists $N \in \mathbb{N}$ such that the following holds. Suppose $\zeta \in \mathcal{E}_{s}$ is at least $N$-times renormalizable. Then the renormalization $\mathcal{R}^{N} \zeta \in \mathcal{E}_{s}$. Further, the map $f_{\zeta}$ is cylinder renormalizable in such a way that its renormalization is $\hat{f}=f_{\mathcal{R}^{N} \zeta}$, which is in $\mathbf{C}_{V}$ with $V \ni U$.

Definition 8.1. For the remainder of the paper fix the value of $N$ as above, and set $\mathbf{M}=\mathbf{C}_{U}^{\mathbb{R}}$. We will call $\hat{f}$ as in Proposition 8.4 the cylinder renormalization of $f_{\zeta}$, and write

$$
\hat{f} \equiv \mathcal{R}_{\mathrm{cyl}} f_{\zeta}
$$

By Proposition 8.1, for every pair $\zeta$ as above, the tranformation $f_{\zeta} \mapsto \mathcal{R}_{\text {cyl }} f_{\zeta}$ extends to an open neighborhood $Y \subset \mathbf{C}_{U}$ as an analytic operator $Y \rightarrow \mathbf{C}_{U}$. We shall call this operator the cylinder renormalization operator.

Proposition 8.5. If $\zeta_{1}, \zeta_{2}$ are at least $N$-times renormalizable elements of $\mathcal{E}_{s}$, and $\zeta_{1} \widetilde{\text { conf }} \zeta_{2}$, then $\mathcal{R}^{N} \zeta_{1} \widetilde{\text { conf }_{f}} \mathcal{R}^{N} \zeta_{2}$. Hence the action of $\mathcal{R}^{N}$ is well-defined on the quotient space $\mathcal{E}_{s} / \widetilde{c o n f}$, and denoting $\iota:[\zeta]_{\text {conf }} \rightarrow \mathbf{C}_{U}^{\mathbb{R}}$, we have

$$
\iota \circ \mathcal{R}^{N}=\mathcal{R}_{c y l} \circ \iota .
$$


Hyperbolicity of the renormalization horseshoe. Now let $\hat{f} \in \mathbf{M}$ be a point in the renormalization horseshoe. That is, $\hat{f}=f_{\zeta}$ for a commuting pair $\zeta$ in the horseshoe $\mathcal{I}$ of $\mathcal{R}$ (see $[$ Ya2 $)$. Set $\rho=\rho(\hat{f})$ and define

$$
\mathcal{D}_{\rho}=\{f \in \mathbf{M}, \text { such that } \rho(f)=\rho\} .
$$

We have:

Theorem 8.6 (Theorem 8.2, YYa3]). There exists an open neighborhood $W \subset \mathbf{M}$ of $\hat{f}$ such that $\mathcal{D}_{\rho} \cap W$ is a smooth submanifold of $\mathbf{M}$ of codimension 1.

Let the hyperplane $T \equiv T_{\hat{f}}\left(\mathcal{D}_{\rho} \cap W\right)$ be the tangent space to this codimension one submanifold at $\hat{f}$, and let $\mathcal{L}$ be the differential of $\mathcal{R}_{\text {cyl }}$ at $\hat{f}$ :

$$
\mathcal{L}=D_{\hat{f}} \mathcal{R}_{\mathrm{c} y l}^{m}: \mathbf{B}_{U}^{\mathbb{R}} \rightarrow \mathbf{B}_{U}^{\mathbb{R}}, \mathcal{L}: T \rightarrow T
$$

Recall that a continuous linear operator on a Banach space is called compact if it maps the closed unit ball of the space onto a compact set. This condition is equivalent to the image of every closed bounded set being compact.

Proposition 8.7 (Prop. 9.1, Ya3]). The operator $\mathcal{L}=D_{\hat{f}} \mathcal{R}_{c y l}^{m}: \mathbf{B}_{U}^{\mathbb{R}} \rightarrow \mathbf{B}_{U}^{\mathbb{R}}$ is compact.

Proposition 8.8. There exists $a<1$ independent of $\hat{f}$ such that the operator $\left.\mathcal{L}\right|_{T}$ is a contraction by $a$, and moreover, the spectral radius $R_{s p}$ of the operator $\left.\mathcal{L}\right|_{T}$ is at most a.

Proof. The spectral theory of compact operators implies that there exist finitely many eigenvalues $\lambda_{i}$ of $\left.\mathcal{L}\right|_{T}$ with $\left|\lambda_{i}\right|=R_{\mathrm{sp}}\left(\left.\mathcal{L}\right|_{T}\right)$. The space $T$ breaks into a direct sum $E^{1} \oplus E^{2}$, where the latter is a finite-dimensional subspace spanned by the generalized eigenvectors of $\lambda_{i}$, and the action of $\left.\mathcal{R}_{\text {cyl }}\right|_{\mathcal{D}_{\rho}}$ near $\hat{f}$ is dominated by the projection of $\left.\mathcal{L}\right|_{T}$ to $E^{2}$. The Rigidity Theorem implies that $\mathcal{R}_{\text {cyl }}$ is a universally geometric contraction in $W \cap \mathcal{D}_{\rho}$ (Corollary 1.2). This implies that $R_{\mathrm{sp}}\left(\left.\mathcal{L}\right|_{T}\right)<a<1$.

Define a cone $\mathcal{C} \in \mathbf{B}_{U}^{\mathbb{R}}$ as follows:

$$
\mathcal{C}_{f}=\left\{v \in \mathbf{B}_{U}^{\mathbb{R}} \text { such that } \inf _{x \in \mathbb{R}} v(x)>0\right\} .
$$

\section{Lemma 8.9.}

(I) The cone $\mathcal{C}$ is renormalization-invariant: $\mathcal{L}: \mathcal{C} \rightarrow \mathcal{C}$,

(II) Moreover, there exists $\alpha>0$ and $k \in \mathbb{N}$ independent of $\hat{f}$ such that for any vector field $v \in \mathcal{C}$

$$
\inf _{x \in \mathbb{R}} D_{\hat{f}} \mathcal{R}_{c y l}^{m k}(v(x))>(1+\alpha) \inf _{x \in \mathbb{R}} v(x)
$$

(III) Finally, there exists $\ell \in \mathbb{N}$ such that if $v \in \mathbf{B}_{U}$ belongs to the closure of $\mathcal{C}$, and $v \neq 0$, then $\mathcal{L}^{\ell}(v) \in \mathcal{C}$. 
Proof. Let $\tilde{f}=\pi^{-1} \circ \hat{f} \circ \pi$. As follows from an elementary computation, if $\tilde{f}_{t}(x)=$ $\tilde{f}(x)+t v(x)+o(t)$, then

$$
\tilde{f}_{t}^{2}(x)=\tilde{f}^{2}(x)+t v_{2}(x)+o(t)=\tilde{f}^{2}(x)+t\left(\tilde{f}^{\prime}(\tilde{f}(x)) v(x)+v(\tilde{f}(x))\right)+o(t),
$$

and more generally, if we write

$$
\tilde{f}_{t}^{n}(x)=\tilde{f}^{n}(x)+t v_{n}(x)+o(t), \text { then } v_{n}(x)=\tilde{f}^{\prime}\left(\tilde{f}^{n-1}(x)\right) v_{n-1}(x)+v\left(\tilde{f}^{n-1}(x)\right)
$$

Let us denote $C_{k} \Subset U$ the fundamental crescent of $\hat{f}$ corresponding to the $k$-th cylinder renormalization $\mathcal{R}_{\text {cyl }}^{k} \hat{f}$, and $J_{k}=C_{k} \cap \mathbb{T}$. Let $\varphi_{k}(x)$ be the corresponding uniformizing coordinate $C_{k} \rightarrow \mathbb{C} / \mathbb{Z}$, and $\Phi_{k}: C_{n} \rightarrow \mathbb{C}$ its lift. In the local chart given by $\varphi_{k}^{-1}$, the $k$-th cylinder renormalization $\mathcal{R}_{\text {cyl }}^{k} \hat{f}$ is represented by the iterate $g_{k}=\hat{f}^{q_{m k}}$ for some $m \in \mathbb{N}$.

To prove the first claim, observe that by (8.2),

$$
\inf _{x \in \mathbb{R}} v_{n}(x) \geq \inf _{x \in \mathbb{R}} v(x)>0 .
$$

The image

$$
D \mathcal{R}_{\mathrm{c} y l} v=\left[\left(\Phi_{k}\right)^{\prime} \circ g_{k} \cdot\left(\left.v_{q_{m k}}\right|_{J_{k}}\right)\right] \circ\left(\Phi_{k}\right)^{-1}
$$

Since $\left(\Phi_{k}\right)^{\prime}>0$, (I) follows.

To prove (II), note that by the real a priori bounds, the sizes of the intervals $J_{k}$ decrease geometrically with $k$. On the other hand, applying the Koebe Distortion Theorem to the conformal extension of $\Phi_{k}$ to $C_{k}$ and its two neighboring iterates, we see that the distortion of $\Phi_{k}$ on the interval $g_{k}\left(J_{k}\right)$ is bounded uniformly in $k$ nd $f$. Hence, there exists $\alpha>0$ and $l \in \mathbb{N}$ independent of $f$ such that for all $k \geq l$

$$
\left.\left(\Phi_{k}\right)^{\prime}\right|_{g_{k}\left(J_{k}\right)}>1+\alpha
$$

and (II) follows from (8.4).

Finally, to see (III), observe that if $v(x) \not \equiv 0$, then there exist $\ell, n \leq q_{m \ell}$ such that $v(x)>0$ for every $x \in f^{n}\left(J_{\ell}\right)$. The claim now follows from (8.2).

We conclude:

Proposition 8.10. There exists $\ell \in \mathbb{N}$ such that the invariant horseshoe of the operator $\mathcal{R}_{\text {cyl }}^{\ell}$ is hyperbolic with one-dimensional unstable direction.

Proof. Let $k, m, \alpha$ be as in the previous lemma, and set $\ell=m k$. In view of Proposition 8.8 we only need to construct the one-dimensional expanding direction of $\mathcal{R}_{\text {cyl }}^{\ell}$. For a periodic point $\hat{f}$ of period $p$ of this operator, the previous lemma implies that $R_{\mathrm{sp}}\left(D_{\hat{f}} \mathcal{R}_{\mathrm{c} y l}^{\ell p}\right)>(1+\alpha)^{p}$. Moreover, since the spectrum of a compact operator is discrete, and every non-zero element of the spectrum is an eigenvalue, the corresponding eigendirections vary continuously with $\hat{f}$. The statement easily follows. 


\section{Concluding Remarks}

Let us conclude by outlining some of the recent developments, and open problems which naturally relate to the results of this paper. Firstly, de Faria and de Melo dFdM1 have introduced a class of rotation numbers $\mathcal{F} \mathcal{M} \subsetneq \mathbb{T} \backslash \mathbb{Q}$ specified by the following conditions: $\rho=\left[r_{0}, r_{1}, \ldots\right] \in \mathcal{F} \mathcal{M}$ if

- $\lim \sup \frac{1}{n} \sum_{j=0}^{n-1} \log r_{j}<\infty$,

- $\lim \frac{1}{n} \log r_{n}=0$,

- $\frac{1}{n} \sum_{j=k}^{k+n-1} \log r_{j} \leq \omega_{\rho}\left(\frac{n}{k}\right)$, for $0<n \leq k$, where $\omega_{\rho}(t)$ is a positive function defined for $t>0$ and such that $t \omega_{\rho}(t) \rightarrow 0$ as $t \rightarrow 0$.

The class $\mathcal{F} \mathcal{M}$ has full Lebesgue measure on the circle, and contains all the rotation numbers of bounded type. de Faria and de Melo proved the following:

Theorem dFdM1 Let $f$ and $g$ be two critical circle mappings of class $C^{3}$ with the same irrational rotation number of class $\mathcal{F} \mathcal{M}$ and the same odd integer order of criticality. Suppose that the uniform distance

$$
\operatorname{dist}_{C^{0}}\left(\mathcal{R}^{n} f, \mathcal{R}^{n} g\right) \rightarrow 0 \text { geometrically fast. }
$$

Then there exists a $C^{1+\alpha}$ diffeomorphism of the circle conjugating $f$ and $g$.

In combination with the main theorem of this paper, this implies:

Corollary. Any two analytic critical circle mappings $f$ and $g$ with the same order of the critical point and the same irrational rotation number $\rho \in \mathcal{F} \mathcal{M}$ are $C^{1+\alpha}$-smoothly conjugate.

de Faria and de Melo also provided examples of smooth maps of unbounded type, which have the same rotation numbers and yet are not $C^{1+\alpha}$ conjugate on the whole circle. The first natural question to ask, given the main result of this paper is:

Question. Does the conjugacy between two analytic critical circle maps with an irrational rotation number, which maps the critical point to the critical point, have the smoothness $C^{1+\alpha}$ on the whole circle?

It is also natural to ask:

Question. Are any two smooth critical circle maps of bounded type $C^{1+\alpha}$ conjugate at the critical point?

The counterexamples constructed by de Faria and de Melo make an essential use of the almost parabolic dynamics due to the unbounded type.

Further, there was a recent announcement by K. Khanin and A. Teplinskiy of the following theorem:

Theorem Khan Let $f$ and $g$ be two smooth critical circle mappings with the same irrational rotation number and the same odd integer order of criticality. Suppose that the 
$C^{2+\epsilon}$-distance

$$
\operatorname{dist}_{C^{2+\epsilon}}\left(\mathcal{R}^{n} f, \mathcal{R}^{n} g\right) \rightarrow 0 \text { geometrically fast. }
$$

Then there exists a $C^{1}$-diffeomorphism of the circle conjugating $f$ and $g$.

In combination with the renormalization convergence result of [Ya4] (which we proved by a different method in this paper), this implies:

Corollary. Any two analytic critical circle mappings $f$ and $g$ with the same order of the critical point and the same irrational rotation number are $C^{1}$-smoothly conjugate.

It is not clear if this result can be generalized to the smooth case. In particular,

Question. Let $f$ and $g$ be two $C^{3}$-smooth critical circle maps with the same irrational rotation number and the same order of the critical point. Is it always true that

$$
\operatorname{dist}\left(\mathcal{R}^{n} f, \mathcal{R}^{n} g\right) \rightarrow 0 \text { geometrically fast? }
$$

Renormalization theory for analytic critical circle maps with a cubic critical point is now complete [Ya3, Ya4, and all the results generalize to the case of a map with an arbitrary odd order $\gamma>1$ of the critical point. However, numerical experiments show that the same kind of renormalization horseshoe exists also for non odd-integer $\gamma>1$. The obvious issues with analyticity preclude from directly extending the existing theory to those cases. This remains therefore an important and challenging problem.

\section{REFERENCES}

[BR] L. Bers \& H.L. Royden. Holomorphic families of injections. Acta Math. 157(1986), 259-286.

[Do] A. Douady. Does a Julia set depend continuously on the polynomial? In Complex dynamical systems: The mathematics behind the Mandelbrot set and Julia sets. ed. R.L. Devaney, Proc. of Symposia in Applied Math., Vol 49, Amer. Math. Soc., 1994, pp. 91-138.

[DH1] A. Douady, J.H. Hubbard. Etude dynamique des polynômes complexes, I-II. Pub. Math. d'Orsay, 1984.

[DH2] A. Douady, J.H. Hubbard. On the dynamics of polynomial-like mappings. Ann. Sci. Éc. Norm. Sup., 18(1985), 287-343.

[dF1] E. de Faria. Proof of universality for critical circle mappings. Thesis, CUNY, 1992.

[dF2] E. de Faria. Asymptotic rigidity of scaling ratios for critical circle mappings. Ergodic Theory Dynam. Systems 19(1999), no. 4, 995-1035.

[dFdM1] E. de Faria and W. de Melo. Rigidity of critical circle mappings I. J. Eur. Math. Soc. (JEMS) 1(1999), no. 4, 339-392.

[dFdM2] E. de Faria and W. de Melo. Rigidity of critical circle mappings II. J. Amer. Math. Soc., 13(2000), no. 2, 343-370.

[Ep1] A. Epstein, Towers of finite type complex analytic maps. PhD Thesis, CUNY, 1993.

[EKT] A. Epstein, L. Keen, C. Tresser. The set of maps $F_{a, b}: x \mapsto x+a+\frac{b}{2 \pi} \sin (2 \pi x)$ with any given rotation interval is contractible. Commun. Math. Phys. 173, 313-333, 1995.

[EY] A. Epstein, M. Yampolsky. The universal parabolic map. IMS at Stony Brook Preprint 2001/04.

[EE] J.-P. Eckmann and H. Epstein. On the existence of fixed points of the composition operator for circle maps. Commun. Math. Phys. 107 (1986), 213-231. 
[FKS] M. Feigenbaum, L. Kadanoff, and S. Shenker. Quasi-periodicity in dissipative systems. A renormalization group analysis. Physica 5D (1982), 370-386.

[He] M. Herman. Conjugaison quasi-symmetrique des homeomorphismes analytiques du cercle a des rotations. Manuscript. 20(2000), 173-229.

[Keen] L. Keen. Dynamics of holomorphic self-maps of $\mathbb{C}^{*}$. In Holomorphic functions and moduli I (ed. D. Drasin et al.) Springer-verlag, New York, 1988.

[Khan] K. Khanin, talk presented at the Kolmogorov-100 conference, Moscow, 2003.

[Lan1] O.E. Lanford. Renormalization group methods for critical circle mappings with general rotation number, VIIIth International Congress on Mathematical Physics (Marseille,1986), World Sci. Publishing, Singapore, 532-536, (1987).

[Lan2] O.E. Lanford. Renormalization group methods for critical circle mappings. Nonlinear evolution and chaotic phenomena, NATO adv. Sci. Inst. Ser. B:Phys.,176, Plenum, New York, 25-36, (1988).

[Lyu2] M. Lyubich. Renormalization ideas in conformal dynamics. Cambridge Seminar "Current Developments in Math.", May 1995. International Press, 1995. Cambridge, MA, 155-184.

[Lyu3] M. Lyubich. Dynamics of quadratic polynomials, I-II. Acta Math., 178(1997), 185-297.

[Lyu4] M. Lyubich. Feigenbaum-Coullet-Tresser Universality and Milnor's Hairiness Conjecture. Ann. of Math. (2) 149(1999), no. 2, 319-420.

[Lyu5] M. Lyubich. Almost every real quadratic map is either regular or stochastic. Annals of Math., to appear.

[LY] M. Lyubich and M.Yampolsky. Dynamics of quadratic polynomials: complex bounds for real maps. Ann. l'Inst. Fourier 47, 4(1997), 1219-1255.

[MP] R.S. MacKay, I.C. Percival. Universal small-scale structure near the boundary of Siegel disks of arbitrary rotation number. Physica 26D(1987), 193-202.

[MSS] R. Mañé, P. Sad, D. Sullivan. On the dynamics of rational maps. Ann. Sci. Éc. Norm. Sup. 16(1983), 193-217.

[McM1] C. McMullen. Complex dynamics and renormalization. Annals of Math. Studies, v.135, Princeton Univ. Press, 1994.

[McM2] C. McMullen. Renormalization and 3-manifolds which fiber over the circle. Annals of Math. Studies, Princeton University Press, 1996.

[Mes] B.D. Mestel. A computer assisted proof of universality for cubic critical maps of the circle with golden mean rotation number. PhD Thesis, University of Warwick, 1985.

[Mil] J. Milnor. Dynamics in one complex variable. Introductory lectures. Friedr. Vieweg \& Sohn, Braunschweig, 1999.

[MvS] W. de Melo \& S. van Strien. One dimensional dynamics. Springer-Verlag, 1993.

[ORSS] S. Ostlund, D. Rand, J. Sethna, E. Siggia, Universal properties of the transition from quasiperiodicity to chaos in dissipative systems. Physica, 8D(1983), 303-342.

[Sul1] D.Sullivan. Quasiconformal homeomorphisms and dynamics, topology and geometry. Proc. ICM86, Berkeley, v. II, 1216-1228.

[Sul2] D.Sullivan. Bounds, quadratic differentials, and renormalization conjectures. AMS Centennial Publications. 2: Mathematics into Twenty-first Century (1992).

[Sh] M. Shishikura. The Hausdorff dimension of the boundary of the Mandelbrot set and Julia sets. Ann. of Math. (2) 147(1998), no. 2, 225-267.

[Sw1] G.Świagtek. Rational rotation numbers for maps of the circle. Commun. Math. Phys. 119(1988), 109-128.

[Ya1] M. Yampolsky. Complex bounds for renormalization of critical circle maps, Erg. Th. E Dyn. Systems. 19(1999), 227-257. 
[Ya2] M. Yampolsky. The attractor of renormalization and rigidity of towers of critical circle maps, Commun. Math. Phys., 218(2001), no. 3, 537-568.

[Ya3] M. Yampolsky. Hyperbolicity of renormalization of critical circle maps. Publ. Math. IHÉS, No. 96 (2002), 1-41

[Ya4] M. Yampolsky. The global horseshoe for the renormalization of critical circle maps. Commun. Math. Phys., 240(2003), 75-96.

[Yoc] J.-C. Yoccoz, Il n'ya pas de contre-example de Denjoy analytique, C.R. Acad. Sci. Paris 298(1984) série I, 141-144. 